\title{
Barreras y facilitadores de acceso a la atención de salud: una revisión sistemática cualitativa
}

\author{
Macarena Hirmas Adauy, ${ }^{1}$ Lucy Poffald Angulo, ${ }^{1}$ \\ Anita María Jasmen Sepúlveda, ${ }^{2}$ Ximena Aguilera Sanhueza, ${ }^{1}$ \\ Iris Delgado Becerra ${ }^{1}$ y Jeanette Vega Morales ${ }^{3}$
}

Forma de citar

Hirmas Adauy M, Poffald Angulo L, Jasmen Sepúlveda AM, Aguilera Sanhueza X, Delgado Becerra I, Vega Morales J. Barreras y facilitadores de acceso a la atención de salud: una revisión sistemática cualitativa. Rev Panam Salud Publica. 2013;33(3):223-9.

RESUMEN Objetivo. Determinar si las barreras y los elementos facilitadores de acceso a la atención de salud son transversales a distintas poblaciones, países y patologías, e identificar en qué etapas del proceso de acceso a la atención sanitaria se presentan con más frecuencia.

Métodos. Revisión sistemática cualitativa de literatura publicada durante el período 2000-2010. Se consultaron seis fuentes internacionales: Fuente Académica, Medline en texto completo, Base de datos académica multidisciplinaria en texto completo (Academic Search Complete), PubMed, SciELO y LILACS. Se aplicaron criterios de valoración científica del Programa CASPe y la declaración STROBE. En paralelo se revisó literatura gris.

Resultados. Se seleccionaron 19 de 1160 resultados de la revisión de artículos científicos, y 8 de 12 documentos de la revisión de literatura gris. Se identificaron 230 barreras y 35 facilitadores en países con diferentes contextos y grados de desarrollo. Las 230 barreras se clasificaron acorde al modelo de Tanahashi: 25 corresponden a la dimensión disponibilidad, 67 a accesibilidad, 87 a aceptabilidad y 51 a contacto. La mayor proporción de barreras correspondió a la dimensión de aceptabilidad y de accesibilidad. Los elementos facilitadores identificados tienen relación con factores personales, relación entre prestadores y usuarios, apoyo social, información sobre la enfermedad y adaptación de los servicios al paciente.

Conclusiones. La identificación de barreras y facilitadores se realiza mayoritariamente en personas que han contactado los sistemas sanitarios y en todas las etapas del proceso de acceso a la atención de salud. Se identificaron pocos estudios orientados a quienes no contactan los servicios. Las barreras y facilitadores identificados están socialmente determinados, y la mayoría son expresión de inequidades sociales que existen en los países y requieren una acción conjunta con otros sectores distintos de salud para ser reducidas o eliminadas.

Palabras clave Acceso a los servicios de salud; atención a la salud; equidad en salud; inequidad social; cobertura universal.

1 Centro de Epidemiología y Políticas de Salud, Facultad de Medicina, Clínica Alemana-Universidad del Desarrollo, Santiago, Chile. La correspondencia se debe dirigir a Macarena Hirmas, mhirmas@udd.cl

2 Biblioteca Biomédica, Facultad de Medicina, Clínica Alemana-Universidad del Desarrollo, Santiago, Chile.

3 En el momento del estudio: Centro de Epidemiología y Políticas de Salud, Facultad de Medicina, Clínica Alemana-Universidad del Desarrollo, Santiago, Chile.
¿Por qué algunas personas toman contacto con los servicios de salud y otras no? ¿Por qué algunos pacientes adhieren a tratamiento y otros no? Los sistemas de salud tienen éxito cuando las personas se mantienen sanas o, en caso de enfermar, tienen acceso a los servicios sanitarios de acuerdo a su necesidad, siguen el tratamiento - limitando el daño o rehabilitándose- y están satisfechas con los servicios que mejoran su calidad de vida y su nivel de salud $(1,2)$.

El nivel de salud está condicionado por los determinantes sociales de la salud (DSS), definidos como "aquellos factores y mecanismos por los cuales las 
condiciones sociales afectan el nivel de salud" (1-4). Los DSS incluyen las características específicas del contexto social por las cuales las condiciones sociales en que la gente vive y trabaja se traducen en efectos sobre la salud (5).

El sistema sanitario es un determinante de la salud en sí mismo; interacciona y puede modificar el efecto de otros determinantes sociales, ya que tiene la responsabilidad de desempeñar una función activa en las condiciones sociales que afectan el nivel de salud, a través del acceso equitativo a los servicios de atención (5).

Los DSS, incluido el sistema de salud, establecen grandes diferencias en el nivel de salud que son inequidades. El concepto de inequidad implica que "las desigualdades son injustas y evitables, es decir pueden ser prevenidas y remediadas" (6). La equidad en salud apunta a que cada persona tenga la misma oportunidad de obtener su máximo potencial en salud y que nadie esté en desventaja para alcanzarlo (3). Para lograr equidad en salud es necesario que exista equidad de acceso a la atención, algo que se logra cuando todas las personas acceden a los servicios de salud y sociales acorde a sus necesidades e independientemente de su capacidad de pago (5).

Distintos modelos han sido utilizados para estudiar el acceso a la atención de salud. Donabedian (7) plantea que el acceso es resultado de variables que van más allá de la disponibilidad de recursos y se centra en aspectos socio-organizacionales y geográficos. Andersen y Aday (8) por su parte sostienen que el acceso a la atención de salud está determinado por las políticas de salud, las características del sistema sanitario y de la población de riesgo, y la utilización de los servicios y la satisfacción del usuario; en este sentido, enfatizan el modo como las políticas de salud determinan las características del sistema y de la población de riesgo, las cuales a su vez influencian la utilización de los servicios y la satisfacción de los usuarios. Andersen y Davidson (9) por su parte identifican cuatro dimensiones de las cuales depende el acceso: características contextuales (sistema de salud, familia y comunidad), características individuales, conductas de salud y resultados. Estos autores consideran que las características contextuales e individuales determinan las conductas de salud y los resultados del proceso de acceso, las cuales retroalimentan a las otras dimensiones.
Según lo postulado por los modelos recién descritos, el acceso a la atención sanitaria sería el resultado del ajuste entre la oferta del sistema de salud y las necesidades de salud expresadas como demanda (10). Tanahashi incorpora este concepto en su "Modelo de Cobertura Efectiva" (11), definiendo cobertura efectiva como la proporción de la población que recibe atención eficaz (donde el rendimiento del servicio entregado se aprecia como satisfactorio en la medida que logra un resultado específico). Plantea que en el proceso de acceso existe una interacción entre aspectos específicos de la provisión de servicios y de la población, que es influenciada tanto por las características del sistema de salud como por los recursos y capacidades de la población para reconocer necesidades y buscar atención. Este modelo es útil para analizar la equidad en el acceso a la atención sanitaria, porque facilita la identificación de grupos específicos con necesidades insatisfechas e identifica las barreras y facilitadores que obstaculizan o favorecen el logro de la cobertura efectiva en cada uno de tales grupos $(10,11)$.

El modelo de Tanahashi considera cuatro etapas en el proceso de acceso para obtener cobertura efectiva: i) disponibilidad, entendida como disponibilidad de servicios del programa o centro de atención, recursos humanos, equipos, insumos, infraestructura e información; ii) accesibilidad, asociada a accesibilidad física como distancia, conectividad, y existencia y tiempo de transporte; organizacional/administrativa, relacionada con requisitos administrativos para la atención, y con la modalidad para obtener horas y horarios de atención, y financiera, relacionada con el costo de transporte, gasto de bolsillo y pérdida de ganancia en el trabajo; iii) aceptabilidad de los servicios, que depende de la percepción de las prestaciones, influenciada por factores sociales, culturales y religiosos, creencias, normas y valores, trato y privacidad, entre otros; iv) contacto con el servicio, entendido como la continuidad de la atención dada por la adaptación de los servicios de salud al paciente y por la calidad de la atención; y v) cobertura efectiva (figura 1) $(10,11)$.

El acceso o abandono de los servicios de salud puede explicarse por elementos facilitadores y obstáculos identificables en las distintas fases del modelo, donde hay grupos sociales -en general las poblaciones más vulnerables- que enfrentan diversas barreras y no tienen contacto con los servicios $(10,12)$.

Varios trabajos han estudiado obstáculos y elementos facilitadores al acceso a los servicios sanitarios en poblaciones, programas y patologías específicas, identificando barreras personales, geográficas, económicas y del propio sistema de salud entre las principales (10, 13). A partir de una revisión de la literatura internacional sobre el tema, los

FIGURA 1. Modelo de evaluación de equidad de acceso y barreras para lograr cobertura de salud universal con equidad

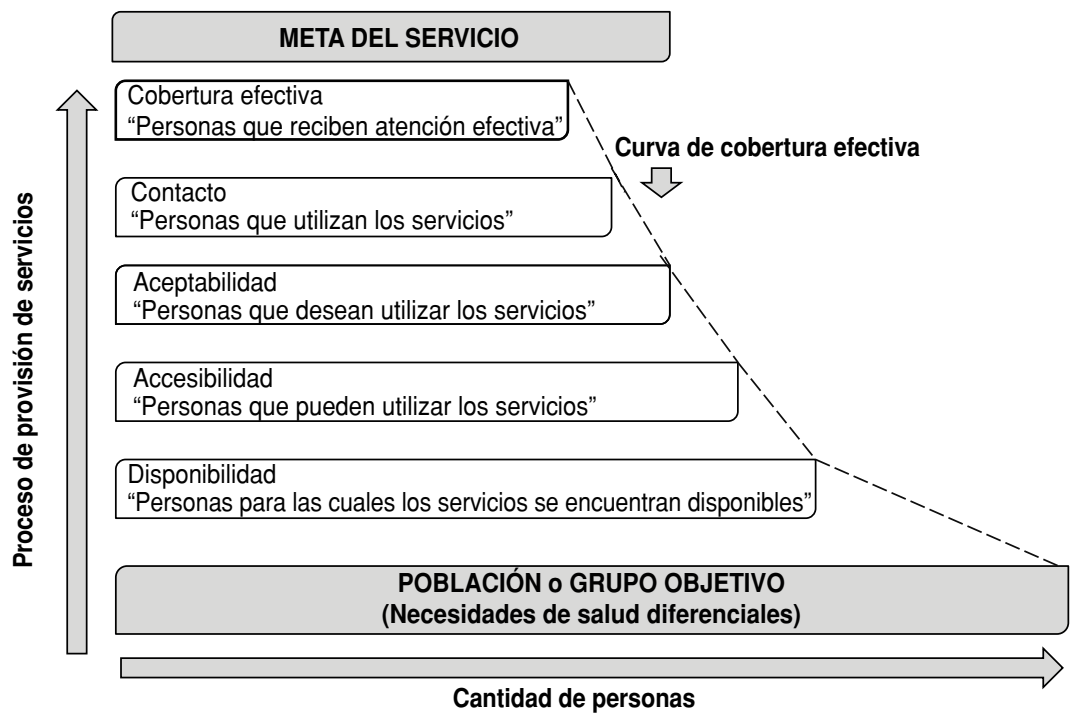

Fuente: Tanahashi T. Health service coverage and its evaluation. Bull World Health Organ. 1978;56(2):295-303. Adaptada y traducida al español por los autores, con autorización. 
objetivos del presente estudio son determinar si las barreras y los elementos salud son transversales a distintas poblaciones, países y patologías, e identificar en qué etapas del proceso de acceso a la atención sanitaria se presentan con más frecuencia.

\section{MATERIAL Y MÉTODOS}

Se realizó una revisión sistemática cualitativa de literatura entre junio y octubre de 2010, planteándose la siguiente pregunta: ¿Cuáles son las barreras y facilitadores de acceso a atención de salud identificadas en la literatura, desde el punto de vista de los usuarios y del personal de salud? Se examinaron artículos publicados - en español e inglés- entre 2000 y 2010 de estudios cuantitativos o cualitativos, de fuente primaria o secundaria, así como literatura gris, que identificaran barreras y facilitadores de acceso a la atención de salud según usuarios y personal de salud.

Una revisión sistemática cualitativa, resume y analiza evidencia en torno a una pregunta de investigación específica los resultados con métodos estadísticos como el metaanálisis (14-18).

Se determinaron palabras clave y limitadores enmarcados bajo el Modelo de Tanahashi, comparándoselos luego con los existentes en la Lista de Términos Médicos (MeSH, por sus siglas en inglés) (cuadro 1). La mayoría no contaba con palabras símiles, ya que corresponden a términos representativos y específicos del campo a estudiar.

Se consultaron tres fuentes internacionales suscritas por la Biblioteca Biomédica facilitadores de acceso a la atención de y estructurada donde no se combinan

de la Facultad de Medicina de la Universidad del Desarrollo, Chile (Fuente Académica, Medline en texto completo y Base de datos académica multidisciplinaria en texto completo [Academic Search Complete]), todas pertenecientes al proveedor EBSCO, y tres fuentes internacionales de acceso libre validado (LILACS, PubMed y SciELO). Para evitar sesgo de publicación, se consultó literatura gris correspondiente a manuales, documentos técnicos e informes de jornadas científicas nacionales e internacionales, así como documentos del Ministerio de Salud de Chile, Organización Mundial de la Salud, el Fondo Commonwealth y de expertos en DSS e inequidades

La búsqueda de artículos científicos se hizo en cada una de las seis bases de datos, por cada palabra clave, y utilizando conectores booleanos para aplicar los filtros definidos (cuadro 1). La secuencia de búsqueda fue: palabra clave (AND) término incluyente (AND) límite de período (NOT) término excluyente. En el caso de la literatura gris, se realizaron consultas a expertos y a fuentes de información indicadas (manuales, documentos técnicos, informes de jornadas y documentos de diferentes organismos).

Dos revisoras examinaron los contenidos en forma paralela e independiente. Los desacuerdos se trataron para lograr un consenso. La valoración de artículos científicos se realizó utilizando listas de chequeo con criterios de evaluación desarrolladas por el Programa de Habilidades en Lectura Crítica (CASPe, por sus siglas en inglés) y la declaración STROBE (siglas en inglés de Fortalecimiento de la comunicación de estudios observacionales en epidemiología) (16, 19, 20).

CUADRO 1. Palabras clave y términos utilizados en la búsqueda de literatura en español e inglés sobre barreras y facilitadores de acceso a la atención de salud publicados en 2000-2010

\begin{tabular}{lll}
\hline \multicolumn{1}{c}{ Palabras clave } & \multicolumn{1}{c}{ Términos incluyentes } & \multicolumn{1}{c}{ Términos excluyentes } \\
\hline Acceso equitativo a salud/Equitable Health Access & Servicios de salud & Legislación \\
Cobertura universal de salud/Universal Coverage & Indicadores & Reforma \\
Adherencia/Adherence & Métodos & Seguros de salud \\
Facilitadores/Facilitators & Instrumentos & Población menor de 15 años \\
Barreras de acceso/Access Barriers & Encuestas & Gasto en salud \\
Barreras de aceptabilidad/Acceptability Barriers & Mediciones & Procedimientos clínicos, \\
farmacológicos
\end{tabular}

En primer lugar, cada revisora identificó de manera independiente los estudios según el tipo de diseño descrito. Luego se aplicaron los criterios de evaluación, asignando a cada estudio si cumplía o no con cada criterio. Para ser aprobado a los fines del presente trabajo, un artículo científico debía cumplir con al menos $80 \%$ de la lista los criterios establecidos, con especial valoración de la metodología.

En estudios de cohortes, caso-control y transversales, la lista de chequeo consideró 22 criterios referidos a título, resumen, introducción, metodología, resultados, discusión, aspectos éticos, financiamiento y conflicto de intereses.

En diseños cualitativos, la lista de chequeo consideró 35 criterios referidos a objetivos, congruencia y adecuación de la metodología a los objetivos, metodología, estrategia de selección de participantes, técnica de recogida de datos, reflexividad del investigador, aspectos éticos, análisis de datos, exposición y aplicabilidad de resultados.

En las revisiones sistemáticas, la lista de chequeo evaluó 22 criterios que correspondieron a tema de la revisión, estrategia de búsqueda, criterios de inclusión, valoración de la calidad de los estudios, resultados, aplicación de resultados y evaluación de beneficios.

En cuanto a la literatura gris, la valoración crítica se basó en dos criterios. El primero correspondió a aquellos documentos que identificaban de forma explícita barreras o facilitadores de acceso a la atención de salud en un territorio y población específicos. El segundo, Correspondió a aquellos que identificaban barreras o facilitadores a partir de modelos teóricos de las dimensiones de acceso a la atención de salud.

\section{RESULTADOS}

De la búsqueda de artículos científicos se obtuvo una bibliografía consolidada de 1160 resultados en formato de resumen. La selección de artículos se realizó en tres fases. En primer lugar, de los 1160 resúmenes se eliminaron aquellos que no cumplieron con los criterios de inclusión, obteniéndose 186 resúmenes. En segundo lugar, se revisó el texto completo de los 186 resúmenes, eliminándose los duplicados y los que no cumplieron con los criterios de inclusión, con un saldo de 68 artículos aprobados. En tercer lugar, se realizó una revisión 
crítica de los artículos seleccionados en la fase anterior, descartándose los que no cumplieron con los criterios de valoración crítica. De esta fase final, se obtuvieron 19 estudios, cuyos contenidos fueron analizados para dar respuesta a la pregunta planteada en el presente trabajo (cuadro 2). De la revisión de literatura gris, se obtuvieron 12 documentos, de los cuales se seleccionaron los ocho que cumplían con los criterios de inclusión (cuadro 3).

\section{Extracción de datos y hallazgos}

De los 19 artículos incluidos, 16 son de fuentes primarias y 3 de fuentes secundarias de información. Como marco muestral, 13 estudios incluyeron exclusivamente a personas que utilizan los servicios de salud, y 6 incluyeron población general usuaria y no usuaria de servicios. Solo cinco estudios incorporaron también a equipos de salud. Seis del total de artículos incluyeron participantes de distinta etnia.

Del total de artículos, 6 corresponden a estudios cuantitativos, 12 a cualitativos y 1 a revisión sistemática. Entre los estudios cuantitativos, tres se realizaron en Canadá, dos en Estados Unidos y uno en Chile. De los estudios cualitativos, cinco se realizaron en Estados Unidos, dos en Canadá, uno en Bolivia, uno en Guatemala, uno en México, uno en Nicaragua y uno en Zambia. La revisión sistemática incluyó 84 estudios, 73 realizados en países desarrollados (56 en Estados Unidos, 3 en Canadá, 3 en Reino Unido, 2 en Francia, 2 en Holanda, 2 en Italia, 1 en Australia, 1 en Bélgica, 1 en Suiza y 2 fueron multinacionales) y 12 en países en desarrollo (4 en Brasil, 1 en Bostwana, 1 en Costa de Marfil, 1 en Costa Rica, 1 en China, 1 en Malawi, 1 en Rumania, 1 en Sudáfrica y 1 en Uganda) (ver material suplementario, cuadro S1). Seis de los 19 artículos se refieren a barreras y faci- litadores de atención en salud general, 4 de terapia antirretroviral, 3 de atención en salud reproductiva, 2 de atención preventiva, 1 de atención preoperatoria, 1 de atención en salud mental, 1 de servicios genéticos y 1 de terapia medicamentosa antihipertensiva.

Los estudios revelan gran variabilidad en las barreras identificadas y proponen distintas taxonomías para clasificarlas. En este caso, se diseñó una matriz con la taxonomía definida por los autores de los estudios para la clasificación de barreras y facilitadores (ver material suplementario, cuadro S2).

Un total de 230 barreras y 35 elementos facilitadores fueron identificados por los usuarios y los equipos de salud. Se contabilizaron todas las barreras que aparecieron en la revisión, independientemente de que fueran las mismas o similares.

Las 230 barreras se clasificaron siguiendo el modelo de Tanahashi: 87 (38\%) correspondieron a la dimensión de aceptabilidad, 67 (29\%) a accesibilidad, $51(22 \%)$ a contacto y $25(11 \%)$ a disponibilidad (figura 2). Considerando el total de las barreras identificadas, las barreras que aparecieron con mayor frecuencia fueron: costo de medicamentos, consultas médicas y exámenes $(8,7 \%)$, temor o vergüenza al atenderse en un servicio de salud $(7,6 \%)$, desconfianza en los equipos de salud y en el tratamiento prescrito $(6,1 \%)$ y estigma social, creencias y mitos $(6,1 \%)$. Dado que el objetivo fue identificar barreras y facilitadores de acceso a la atención, y no la proporción de la población mencionada, no se incorporó la dimensión "cobertura efectiva" del modelo de Tanahashi (figura 2).

De los cinco estudios que incluyeron como sujetos a equipos de salud, solo cuatro identificaron las barreras percibidas por el equipo, principalmente las que corresponden a las dimensiones de disponibilidad y contacto.

CUADRO 2. Resultados de la búsqueda de artículos científicos sobre barreras y facilitadores de acceso a la atención de salud publicados en 2000-2010

\begin{tabular}{lcccc}
\hline \multicolumn{1}{c}{ Términos utilizados } & Encontrados & $\begin{array}{c}\text { Separados } \\
\text { para revisión }\end{array}$ & Aprobados & Incluidos \\
\hline Acceso equitativo a salud & 240 & 38 & 9 & 3 \\
Cobertura universal de salud & 236 & 9 & 4 & 1 \\
Barreras de acceso & 254 & 65 & 31 & 8 \\
Barreras de aceptabilidad & 72 & 12 & 1 & 0 \\
Barreras de disponibilidad & 159 & 36 & 8 & 1 \\
Barreras de contacto & 180 & 21 & 5 & 2 \\
Adherencia/Barreras/Facilitadores & 19 & 5 & 68 & 19 \\
Total & 1160 & 186 & & \\
\hline
\end{tabular}

CUADRO 3. Resultados de la búsqueda de literatura gris sobre barreras y facilitadores de acceso a la atención de salud publicada en 2000-2010

\begin{tabular}{lcc}
\hline Tipo de publicación & Total & Incluidos \\
\hline Manual de referencia & 1 & 1 \\
Documento técnico & 4 & 3 \\
Aporte de expertos & 2 & 1 \\
Literatura de jornadas & 5 & 3 \\
científicas & 12 & 8 \\
Total & \\
\hline
\end{tabular}

a Consulta a expertos en el tema y revisión de literatura específica sugerida por ellos.

En relación con los 35 elementos facilitadores identificados, destacaron: contar con redes de apoyo social (26\%), el valor asignado a la atención de salud para reducir riesgos y complicaciones (14\%), adaptación de los servicios al paciente y programas de manejo de la enfermedad (11\%), comunicación médico-paciente y calidad de la relación entre ambos (9\%), aceptación del diagnóstico (9\%), contar con recordatorios para el tratamiento (9\%) y confianza en los tratamientos (6\%). Estos factores corresponden a $84 \%$ de los facilitadores.

De los ocho documentos incluidos de literatura gris, tres fueron lineamientos programáticos del Ministerio de Salud de Chile en equidad de acceso a la atención de salud, uno proveniente de Estados Unidos compara el sistema de salud con seis países desarrollados, dos presentaciones de jornadas científicas realizadas en África, una revisión sistemática que resume ocho estudios de tres países y un manual de referencia desarrollado por la Organización Mundial de la Salud y la Red Regional para la Equidad en Salud en África (ver material suplementario, cuadro S3).

\section{DISCUSIÓN}

Los hallazgos revelan que la mayoría de los trabajos sobre barreras y facilitadores en la atención de salud han tenido lugar en países desarrollados, corresponden a estudios cualitativos de fuentes primarias y se han realizado con personas usuarias de servicios de salud. Es importante destacar que los estudios cualitativos generaron un espectro más amplio y profundo de barreras y elementos facilitadores que los cuantitativos.

Las barreras se presentan en todos los contextos de desarrollo social y económico estudiados, y en distintos esquemas de financiamiento y aseguramiento 
FIGURA 2. Principales barreras identificadas por dimensión según el Modelo de Tanahashi

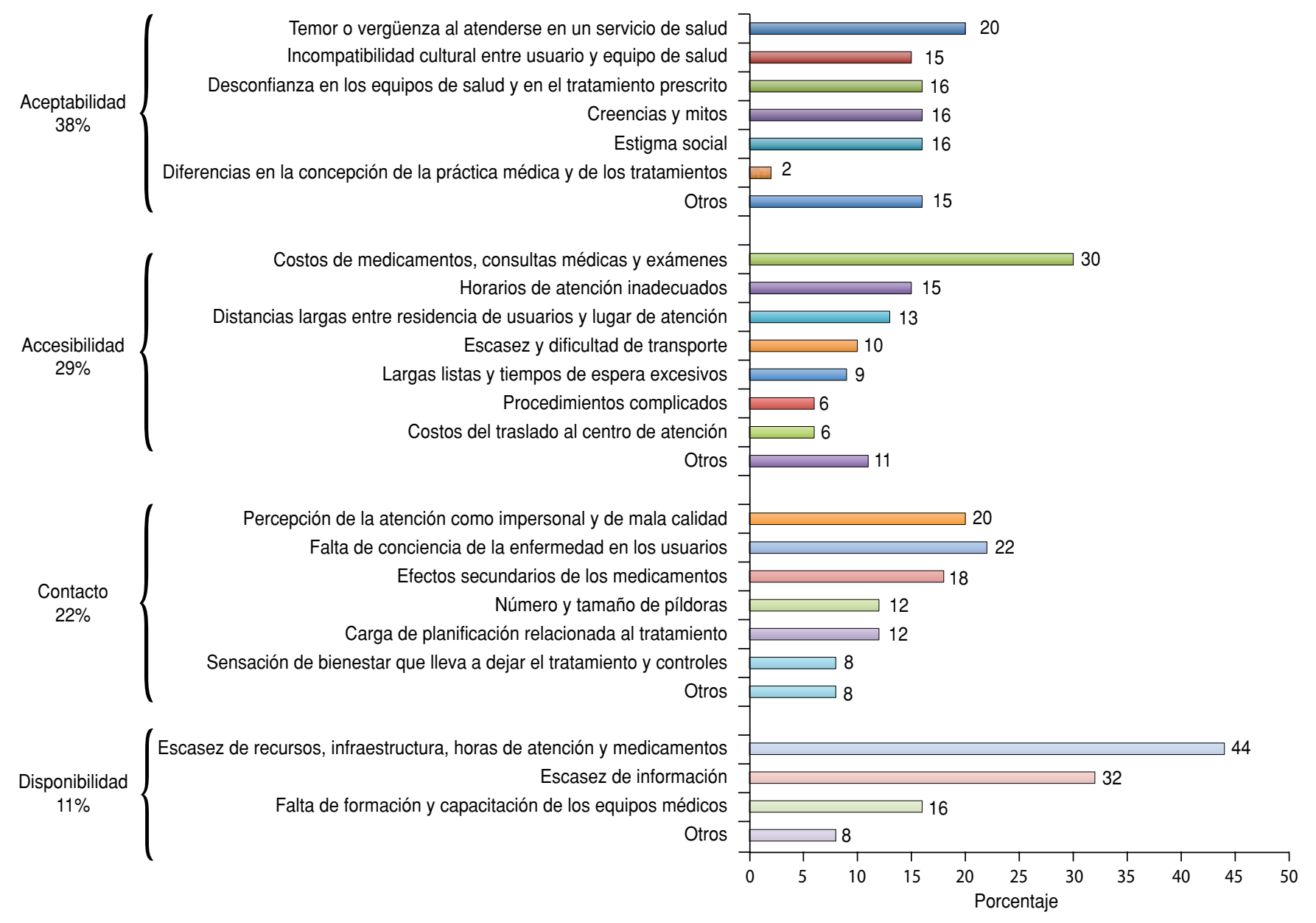

de salud, incluso en países donde existe cobertura universal. Estos hallazgos son coincidentes con los resultados de otros estudios referidos a accesibilidad (10).

Se observó que las barreras que aparecieron con mayor frecuencia fueron: Costo de medicamentos, consultas médicas y exámenes; Temor o vergüenza al atenderse en un servicio de salud; Desconfianza en los equipos de salud y en el tratamiento prescrito, y Estigma social, creencias y mitos $(6,1 \%)$. Los elementos facilitadores más frecuentes fueron Contar con redes de apoyo social; El valor asignado a la atención de salud para reducir riesgos y complicaciones, y Adaptación de los servicios al paciente y programas de manejo de la enfermedad.

En distintos contextos hay similitud en cuanto a que las barreras identificadas son más frecuentes en personas que están en la parte baja de la jerarquía social en la comunidad a la que pertenecen. Esta jerarquía se manifiesta por género o etnia; por posición socioeconómica (según ingreso, ocupación y escolaridad), y por creencias y estilos de vida socialmente determinados. Estos resultados concuerdan con los obtenidos en otras investigaciones (10).

Los estudios y demás documentos examinados no realizan análisis comparativos de las barreras entre los distintos actores involucrados en el proceso de acceso a atención de salud. Sin embargo, sí reportan que los usuarios perciben con mayor frecuencia barreras en las dimensiones de aceptabilidad y accesibilidad, mientras que los equipos de salud identifican con mayor frecuencia barreras relacionadas con disponibilidad y contacto. Esto podría explicarse porque la mayoría de los estudios analizados se realizó con usuarios de servicios que ya tomaron contacto con el sistema, y por tanto la disponibilidad a los servicios no constituye una barrera frecuente. Por su parte los equipos de salud, al tener ma- yor conocimiento de la cobertura real de sus servicios y necesidades de su población, identifican con mayor frecuencia barreras de disponibilidad.

Según Tanahashi, para alcanzar la cobertura efectiva con equidad, las barreras deben ser identificadas en dos direcciones: identificando a quienes no acceden o acceden con mayor dificultad, e identificando en qué etapas del proceso tales barreras tienen lugar con mayor frecuencia, y así dirigir las intervenciones a los "cuellos de botella" específicos. Es posible ordenar en términos del tipo de determinantes y distintas fases del análisis de equidad, y de este modo identificar en cada programa de salud las barreras de acceso y los determinantes sociales específicos en tales programas, y al mismo tiempo identificar barreras comunes a todo el sistema de salud o a un conjunto de programas (21).

Un elemento crucial de la presente revisión sistemática es haber develado 
que en la mayoría de los estudios la identificación de barreras y elementos facilitadores se realiza mayoritariamente $(68 \%)$ en personas que han contactado los sistemas de atención de salud, en atención preventiva, de diagnóstico o tratamiento. Se encontraron pocos estudios que hayan tratado las barreras que enfrentan quienes no contactan los sistemas de atención de salud.

Si se aplica la Ley de atención inversa de Tudor, lo más probable es que quienes no contactan a los sistemas de atención sean grupos más vulnerables que quienes sí los contactan. Tal vulnerabilidad estaría asociada, entre otras razones, a la condición social, económica y cultural de estas poblaciones, motivando que las barreras que enfrentan sean más frecuentes y de mayor complejidad (22). Estos son precisamente los grupos de especial interés para investigar en el marco de una agenda global que avance hacia un acceso a la salud con cobertura universal y equidad. Dicha meta implica que hay que asignar prioridad a la realización de estudios que identifiquen barreras y facilitadores en la población general, comparando a quienes acceden a la atención de salud con quienes no logran contactarse con estos servicios.

Por otra parte, futuros análisis de las dimensiones del modelo de Tanahashi deberían involucrar a los distintos actores del proceso de acceso a la atención de salud, con mayor énfasis en los usuarios. Desde la visión de los prestadores, cuando los servicios están disponibles son los usua- rios quienes tienen barreras para accederlos; no obstante, las barreras de acceso están dadas muchas veces por los prestadores que no se ajustan a las necesidades y las posibilidades reales de accesibilidad de las personas, ni toman en cuenta sus condiciones diferenciales de vida y de trabajo que determinan el acceso a los servicios.

Vale señalar que el presente trabajo no está exento de limitaciones. Una de ellas se refiere a los criterios de inclusión, con especial mención al idioma, ya que excluyó la literatura en portugués, restringiendo así el campo de búsqueda y la diversidad de los estudios obtenidos. La otra limitación tiene que ver con las restricciones de accesibilidad que impidieron agotar la búsqueda de la literatura gris.

\section{Conclusiones}

Se constató que la mayoría de los estudios de identificación de barreras y de elementos facilitadores de acceso a la atención de salud han sido llevados a cabo en países desarrollados, corresponden a estudios cualitativos de fuentes primarias y se han realizado con personas usuarias de servicios de salud. Las barreras y los facilitadores se presentan en países de distinto grado de desarrollo social y económico. Al analizar las barreras según el modelo de Tanahashi, se identifican barreras en todas las dimensiones del modelo.

La identificación de barreras y de elementos facilitadores en el acceso a la atención de salud contribuye a un mo- nitoreo de la equidad en salud más efectivo y es un paso imprescindible para mejorar el acceso de toda la población y reducir las inequidades sociales y de salud. El modelo de Tanahashi permite sistematizar barreras y facilitadores en dimensiones específicas, que al volcarse en un instrumento de chequeo podría resultar valioso al momento de evaluar el acceso a la atención de salud.

El presente estudio confirma la necesidad de diseñar y ejecutar políticas sanitarias participativas e intersectoriales, que reduzcan o eliminen las barreras que son responsabilidad de sectores distintos al sector salud, con el fin de lograr el acceso equitativo a la atención de salud. A su vez, es recomendable que los prestadores de salud adapten la provisión de servicios a las necesidades específicas de su población, diseñen e implementen estrategias de búsqueda de grupos que no contactan al sistema de salud e incorporen en la planificación de servicios los elementos facilitadores identificados.

En continuidad con esta línea de investigación, se requiere identificar barreras y facilitadores de la población que no toma contacto con los servicios de salud y determinar específicamente cuáles son los grupos excluidos de los sistemas de atención.

Financiamiento. El presente trabajo fue financiado por la Fundación Rockefeller, a través del proyecto N. ${ }^{\circ} 2340031$, Universidad del Desarrollo, Chile.

Conflictos de interés. Ninguno.

\section{REFERENCIAS}

1. Lalonde M. A New Perspective on the Health of Canadians: a working document. Canada, Minister of National Health and Welfare; 1981. Disponible en: http://www.hc-sc. gc.ca/hcs-sss/com/fed/lalonde-eng.php Acceso el 23 de enero de 2013.

2. Organización Mundial de la Salud. Subsanar las desigualdades en una generación: alcanzar la equidad sanitaria actuando sobre los determinantes sociales de la salud. Informe final de la Comisión sobre Determinantes Sociales de la Salud. Ginebra: OMS, Comisión sobre Determinantes Sociales de la Salud; 2008.

3. Álvarez Pérez A, García Fariña A, Bonet Gorbea M. Pautas conceptuales y metodológicas para explicar los determinantes de los niveles de salud en Cuba. Rev Cub Salud Pública. 2007. Disponible en: http://scielo.sld.cu/ scielo.php?script=sci_arttext\&pid=S0864$34662007000200013 \& \operatorname{lng}=e s \& n r m=i s o$ Acceso el 23 de enero de 2013.
4. Hosseinpoor AR, Van Doorslaer E, Speybroeck N, Naghavi M, Mohammad K, Majdzadeh R, et al. Decomposing socioeconomic inequality in infant mortality in Iran. Int J Epidemiol. 2006;35(5):1211-9.

5. Vega J, Solar O, Irwin A. Equidad y determinantes sociales de la salud: conceptos básicos, mecanismos de producción y alternativas para la acción. En: Jadue L, Fabiola M, eds. Determinantes sociales de la salud en Chile. En la perspectiva de la equidad. Chile: Iniciativa Chilena de Equidad en Salud (ICES); 2005. Pp. 9-18.

6. Whitehead M. The concepts and principles of equity. Health Promot Int. 1991;6(3):217-28.

7. Donabedian A. Aspects of Medical Care Administration. Cambridge: Harvard University Press; 1973.

8. Aday LA, Andersen R. A Framework for the Study of Access to Medical Care. Health Serv Res. 1974;9(3):208-20.
9. Andersen RM, Davidson PL. Improving access to care in America: Individual and contextual factor. In: Andersen RM, Rice TH, Kominski GF, eds. Changing the American Health Care System: Key Issues in Health Services Policy and Management. 3. ${ }^{\mathrm{a}}$ ed. San Francisco, CA: Jossey-Bass; 2007. Pp. 3-31.

10. Frenz $\mathrm{P}$, Vega J. Universal health coverage with equity: what we know, don't know, and need to know. Backgroung paper for the global symposium on health systems research, 2010. Disponible en: http:/ /www. ops-oms.org.pa/drupal/SCMDSS/2\%20 WCSDH \%20Discussion \%20Paper $\% 20$ resources $/ 3 \% 20$ Health\%20sector/Universal_Health_Coverage_With_Equity_2010. pdf Acceso el 23 de enero de 2013.

11. Tanahashi T. Health service coverage and its evaluation. Bull World Health Organ. 1978;56(2):295-303. 
12. World Health Organization, Regional Network for Equity in Health in East and Southern Africa. A toolkit for monitoring and evaluating equity in access and health systems strengthening in AIDS treatment programmes: concepts, models, methods and indicators, 2010.

13. Gulliford M, Figueroa-Munoz J, Morgan M, Hughes D, Gibson B, Beech R, et al. What does "access to health care" mean? J Health Serv Res Policy. 2002;7(3):186-8.

14. Letelier LM, Manríquez J, Rada G. Revisiones sistemáticas y meta análisis: ¿son la mejor evidencia? Rev Med Chil. 2005;133:246-9.

15. Gisbert JP, Bonfill X. ¿Cómo realizar, evaluar y utilizar revisiones sistemáticas y meta análisis? Gastroenterol Hepatol. 2004;27(3):129-49.

16. Higgins JPT, Green S. Cochrane Handbook for Systematic Reviews of Interventions. Version 5.1.0. (updated March 2011). The Co- chrane Collaboration, 2011. Disponible en: www.cochrane-handbook.org Acceso el 23 de enero de 2013.

17. Green B, Johnson C, Adams A. Writing narrative literature reviews for peer-reviewed journals: secrets of the trades. J Chiropr Med. 2006;5(3):101-17.

18. Noyes J, Popay J. Directly observed therapy and tuberculosis: how can a systematic review of qualitative research contribute to improving services? A qualitative metasynthesis. J Adv Nurs. 2007;57:227-43.

19. Critical Appraisal Skills Programme (CASP) Materiales CASPe, Parrillas para lectura crítica. Disponible en: http://redcaspe.org/ drupal/?q=node/29 Acceso el 23 de enero de 2013.

20. Strengthening the Reporting of Observational Studies in Epidemiology (STROBE Statement). STROBE checklists Disponible en: http://www.strobe-statement.org/index. php?id=available-checklists

21. Solar O. Experiencia y proyecciones del proceso de revisión de programas en Chile. En: II Seminario: Estrategia para la integración de los determinantes sociales y equidad en los programas de salud del Ministerio de Salud de Chile. 26-28 de noviembre, 2008.

22. Tudor J. The inverse care law. Lancet. 1971; 1(7696):405-12.

Manuscrito recibido el 18 de enero de 2012. Aceptado para publicación, tras revisión, el 4 de enero de 2013.
ABSTRACT

Health care access barriers and facilitators: a qualitative systematic review

Key words
Objective. To determine whether health care access barriers and facilitators cut across different populations, countries, and pathologies, and if so, at which stages of health care access they occur most frequently.

Methods. A qualitative systematic review of literature published between 2000 and 2010 was undertaken drawing on six international sources: Fuente Académica, MEDLINE (full-text), Academic Search Complete (a full-text multidisciplinary academic database), PubMed, SciELO, and LILACS. Scientific appraisal guidelines from the Critical Appraisal Skills Programme Español (CASPe) and Strengthening the Reporting of Observational Studies in Epidemiology (STROBE) were applied. Gray literature was also reviewed.

Results. From the review of scientific literature, 19 of 1160 articles and 8 of 12 gray literature documents were selected. A total of 230 barriers and 35 facilitators were identified in countries with different contexts and degrees of development. The 230 barriers were classified according to the Tanahashi framework: 25 corresponded to availability, 67 to access, 87 to acceptability, and 51 to contact. Most of the barriers were related to acceptability and access. The facilitating elements that were identified had to do with personal factors, the provider-client relationship, social support, knowledge about diseases, and adaptation of the services to patients.

Conclusions. The barriers and facilitators were seen mostly in people who initiated contact with the health systems, and they occurred at all stages of health care access. Only a few of the studies looked at people who did not initiate contact with the health services. The barriers and facilitators identified were socially determined and largely a reflection of existing social inequities in the countries. To reduce or eliminate them, joint action with other non-health sectors will be necessary.

Health services accessibility; health care (public health); equity in health; social inequity; universal coverage. 
REVISTA

PANAMERICANA

DE SALUD PÚBLICA
PAN AMERICAN

JOURNAL OF

PUBLIC HEALTH

Material suplementario / Supplementary material / Material supplementar

Material suplementario del artículo:

Hirmas Adauy M, Poffald Angulo L, Jasmen Sepúlveda AM, Aguilera Sanhueza X, Delgado Becerra I, Vega Morales J. Barreras y facilitadores de acceso a la atención de salud: una revisión sistemática cualitativa. 2013;33(3):223-9.

Este material forma parte del artículo presentado originalmente y ha sido sometido a revisión externa. Se presenta tal como ha sido remitido por los autores. 


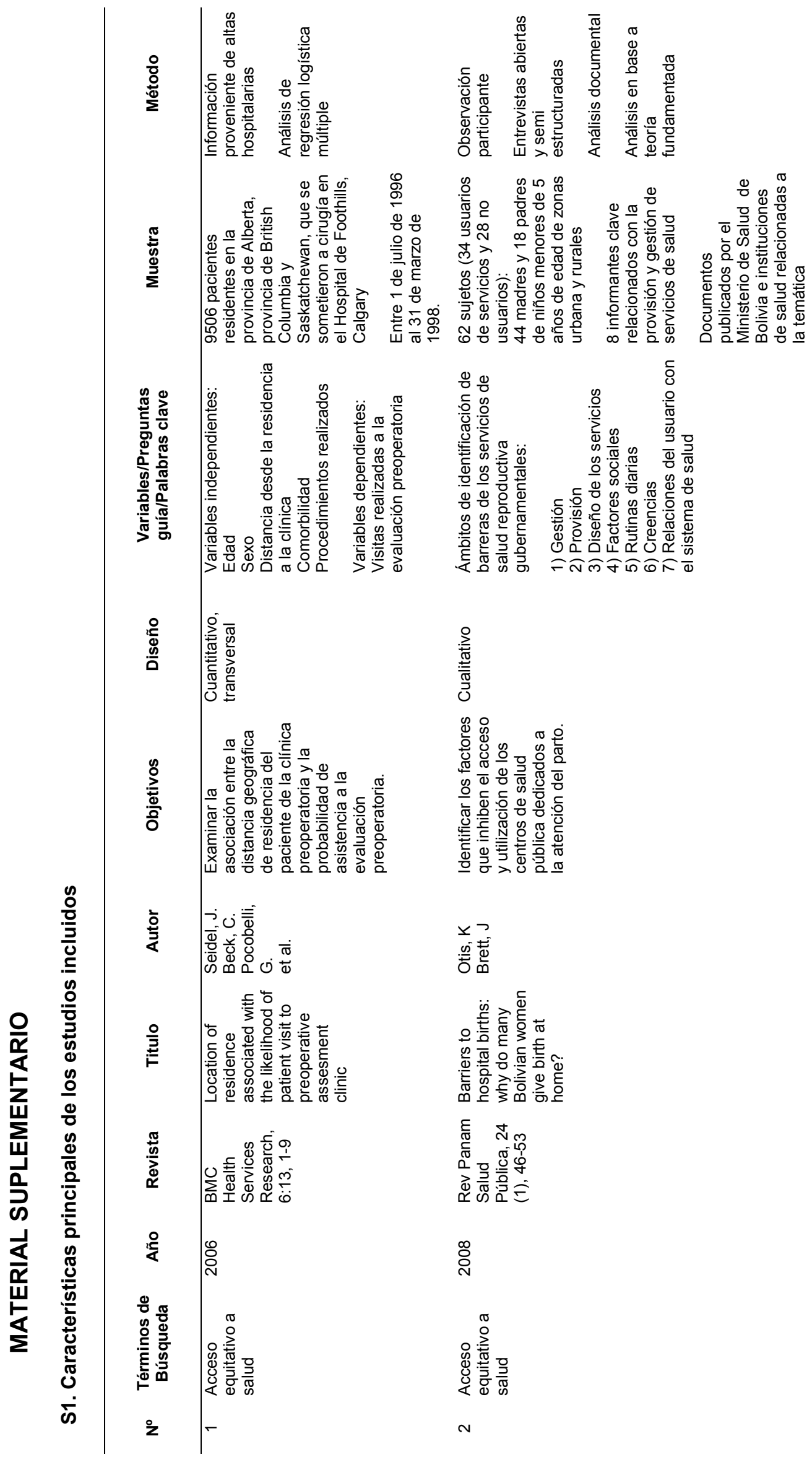




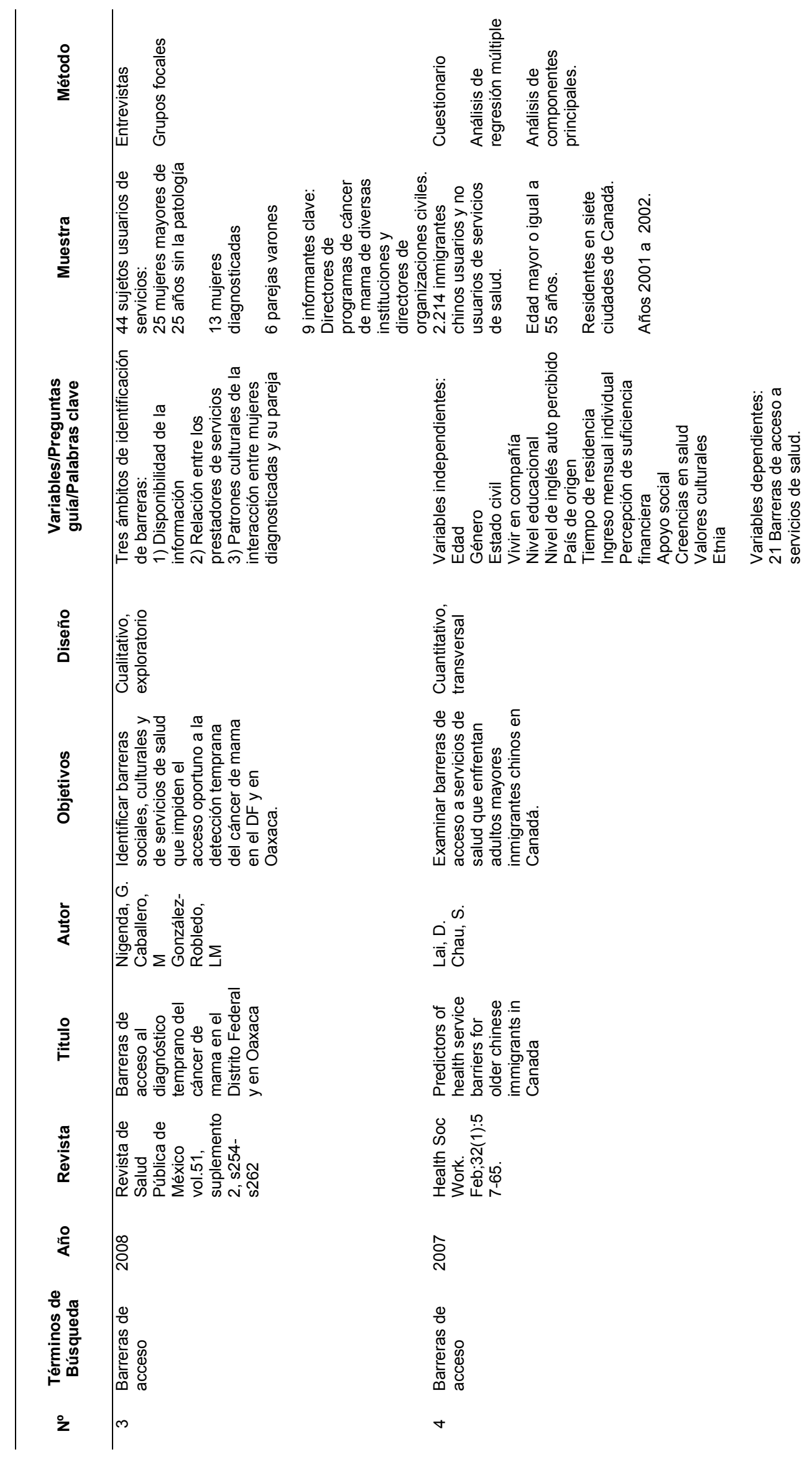




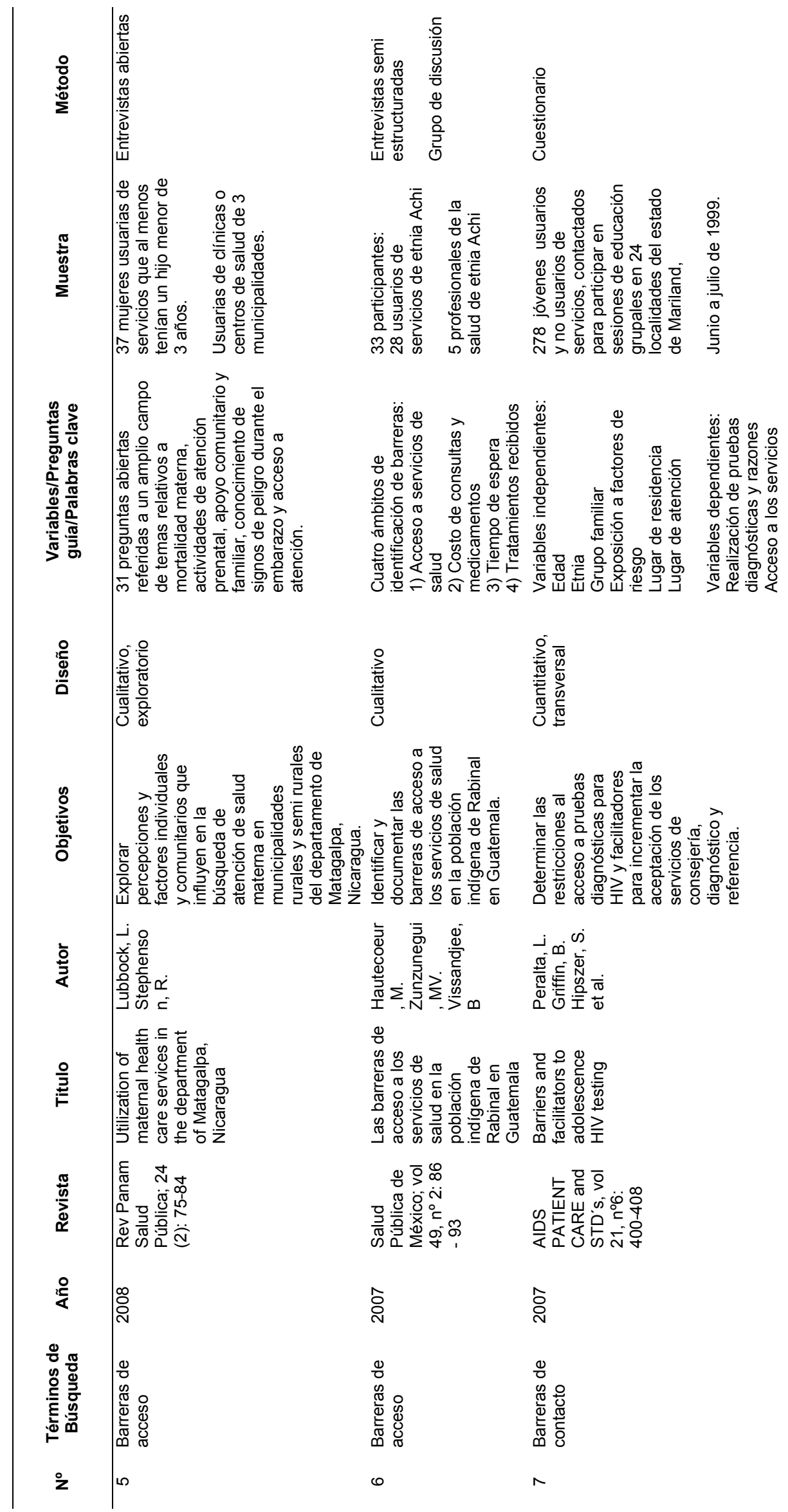




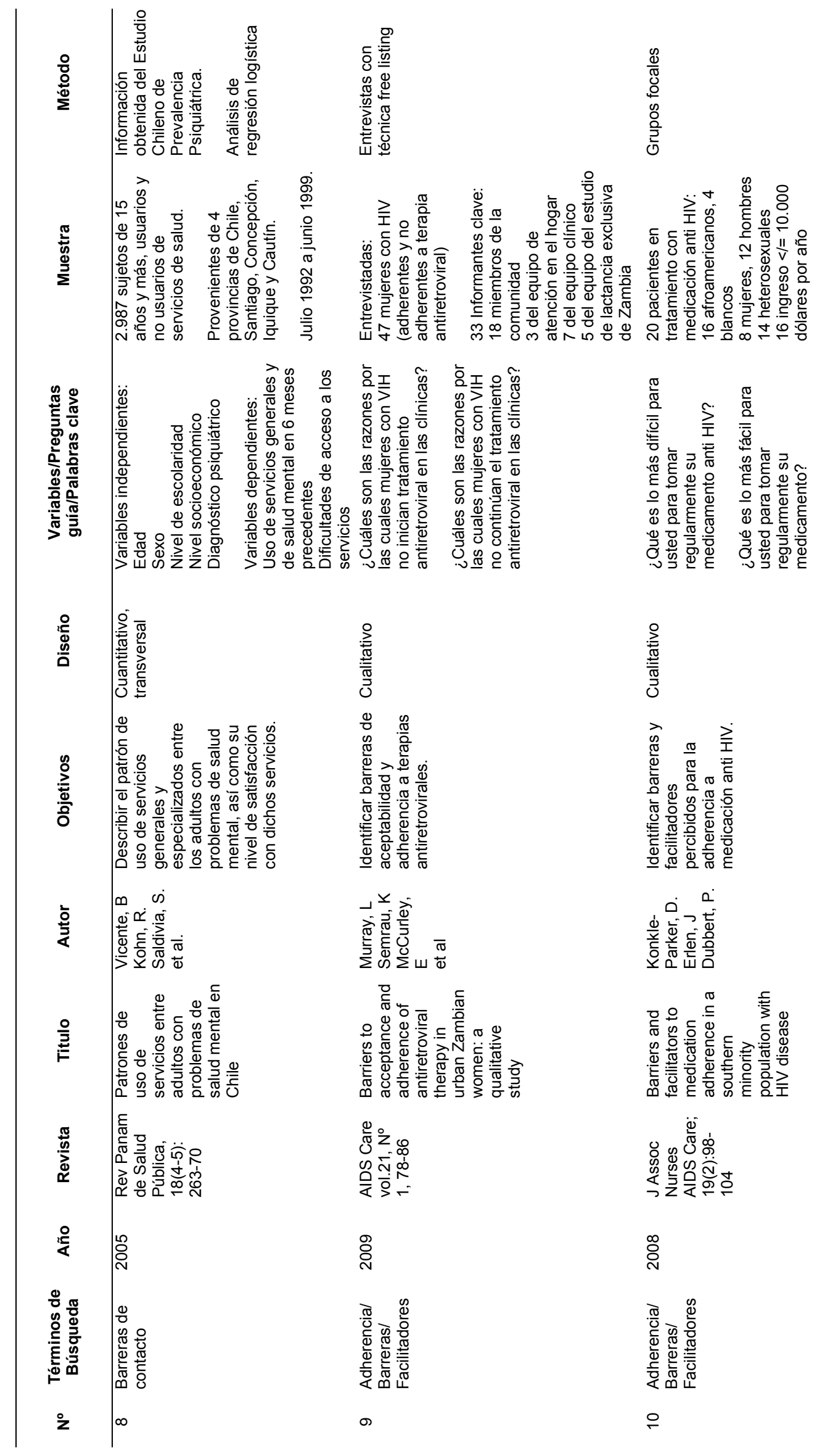




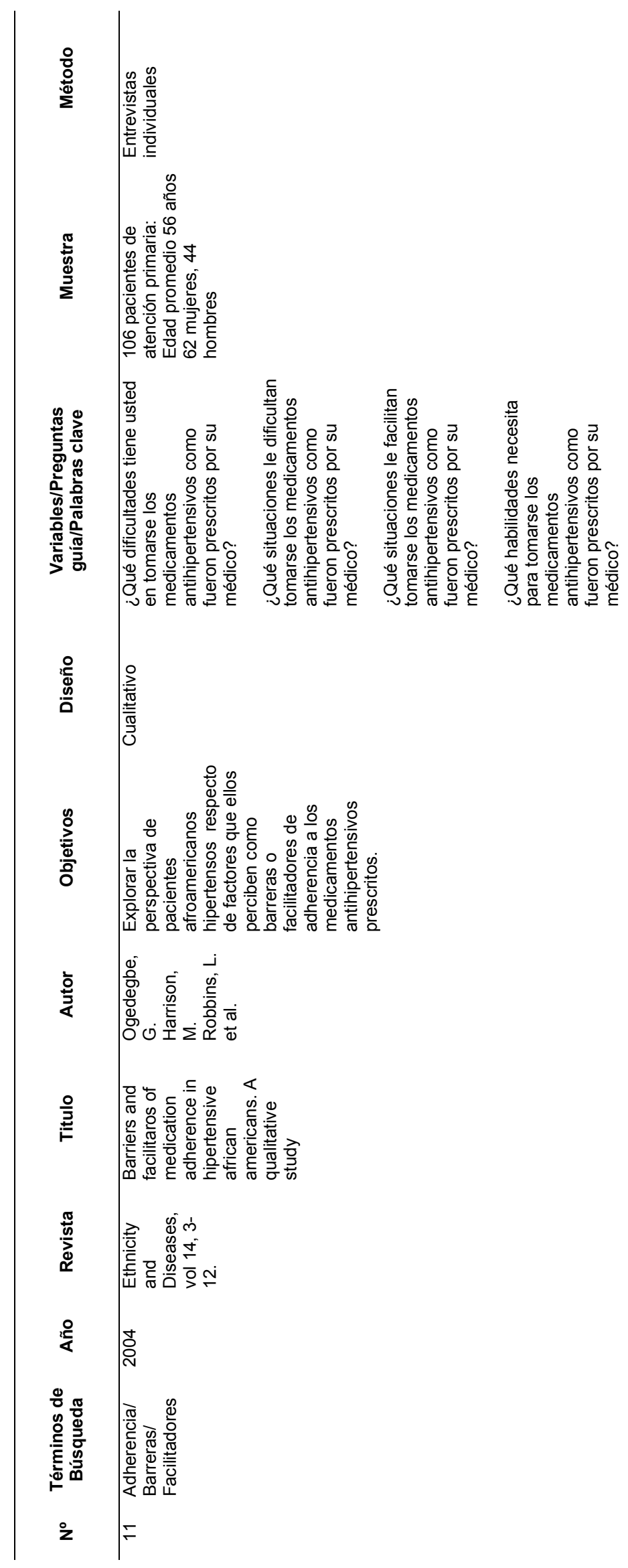




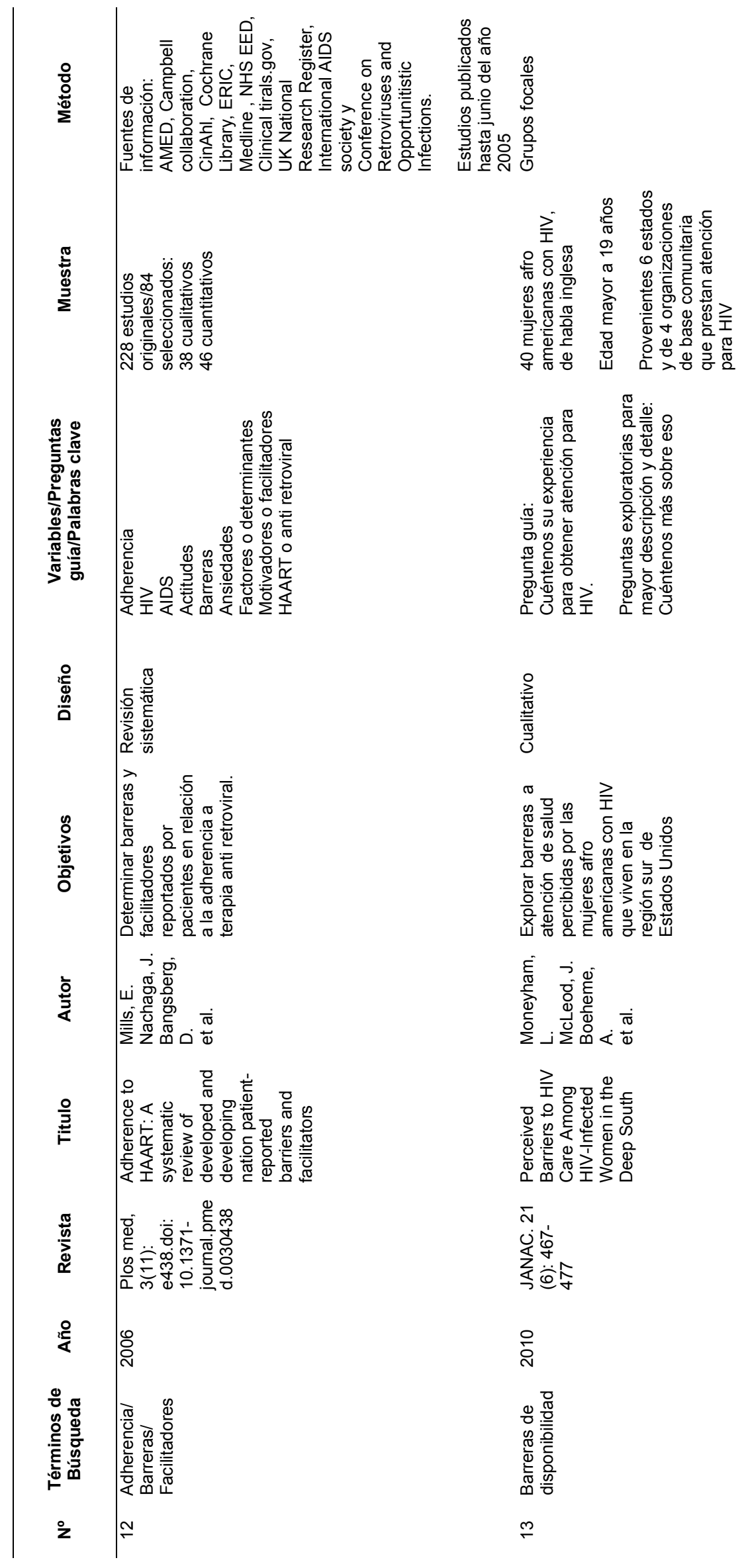




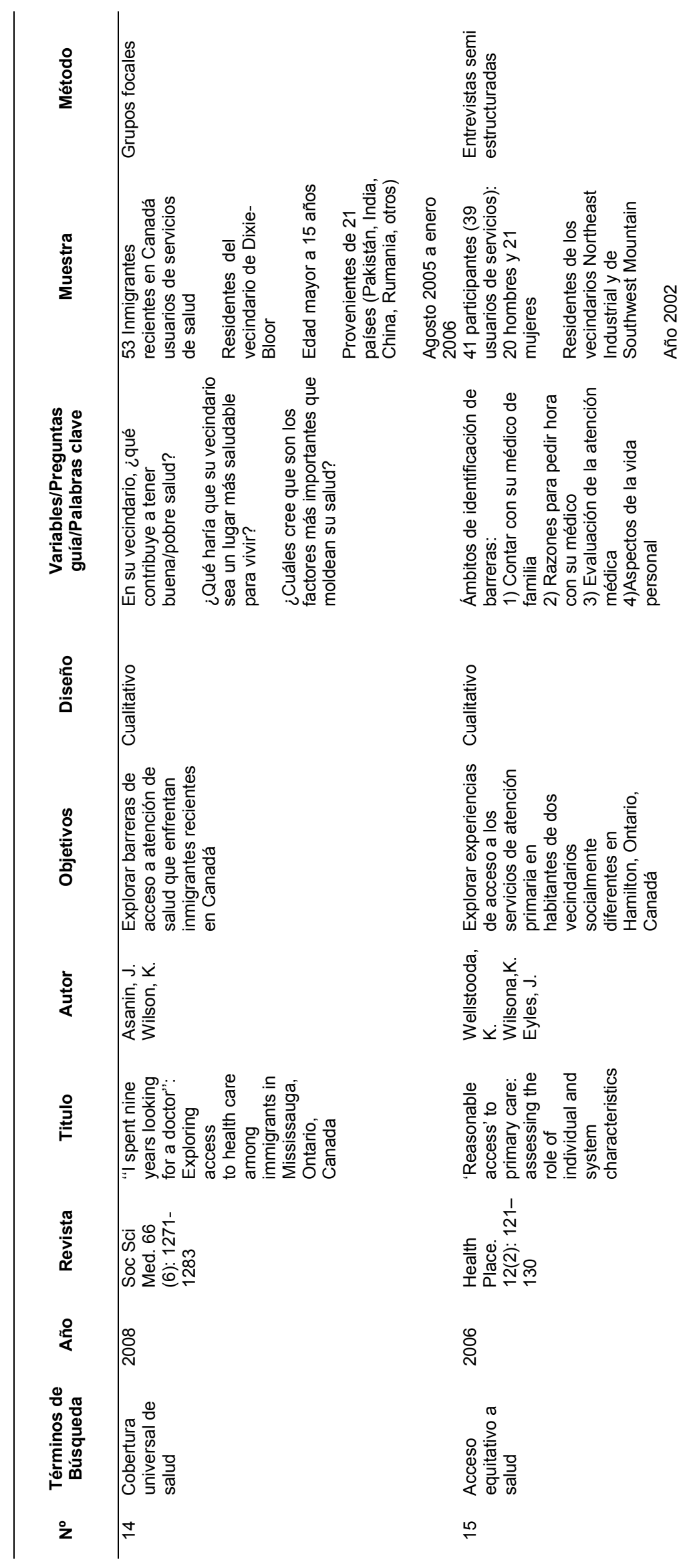




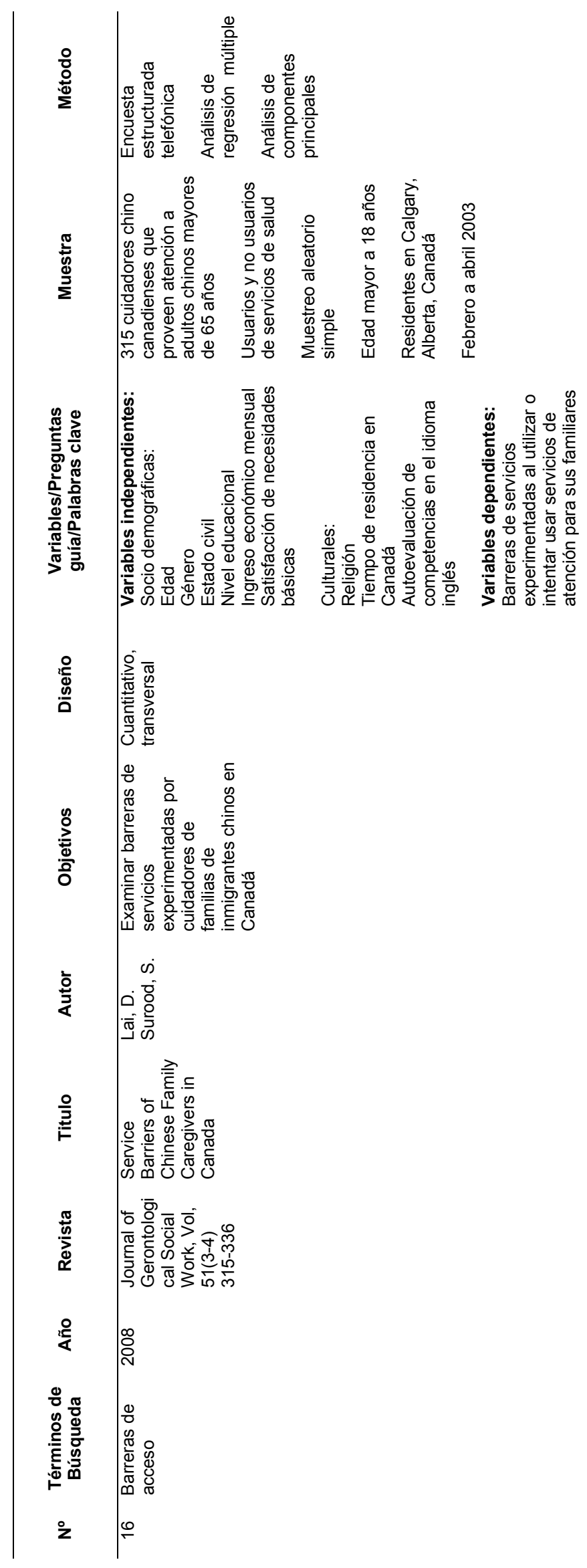




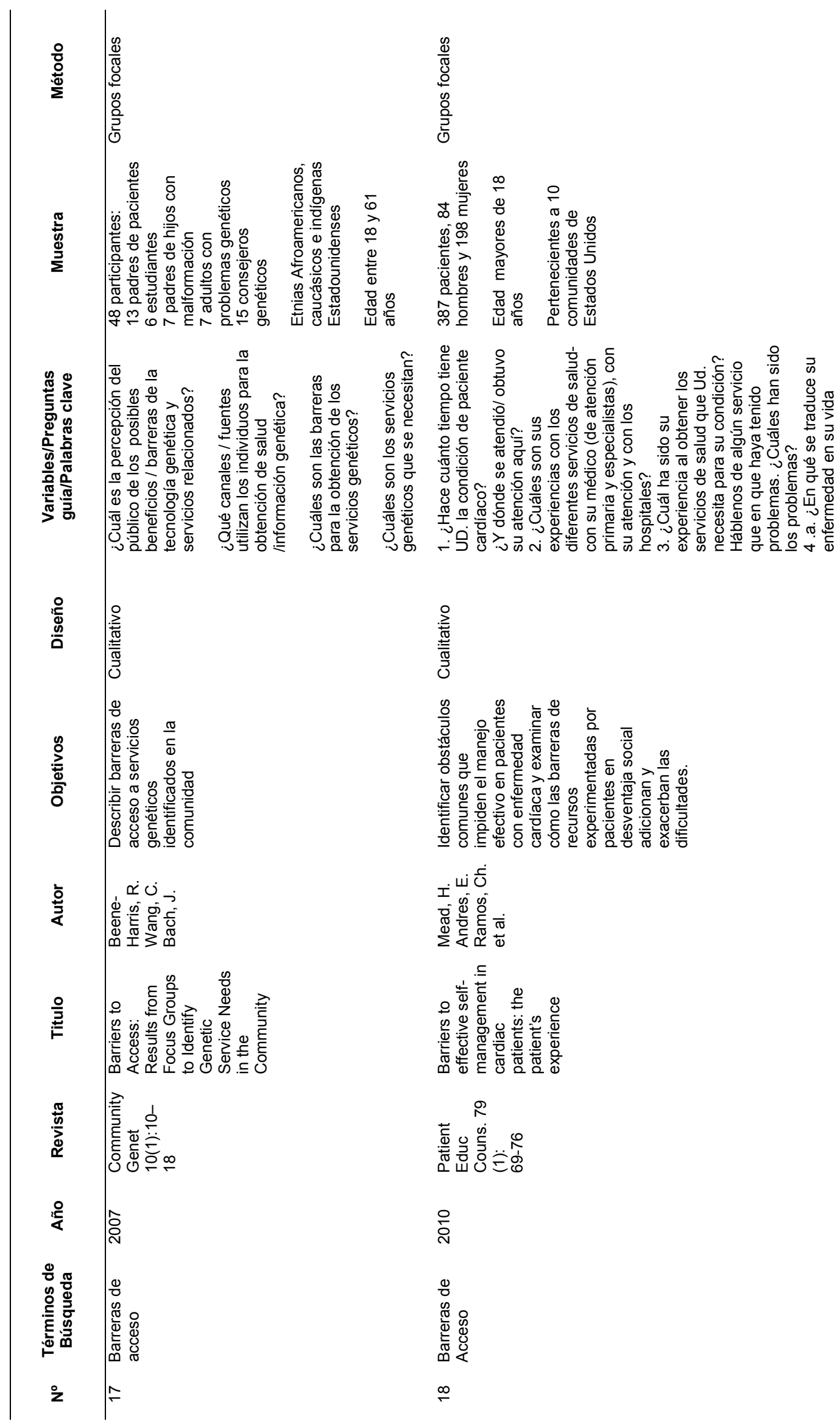




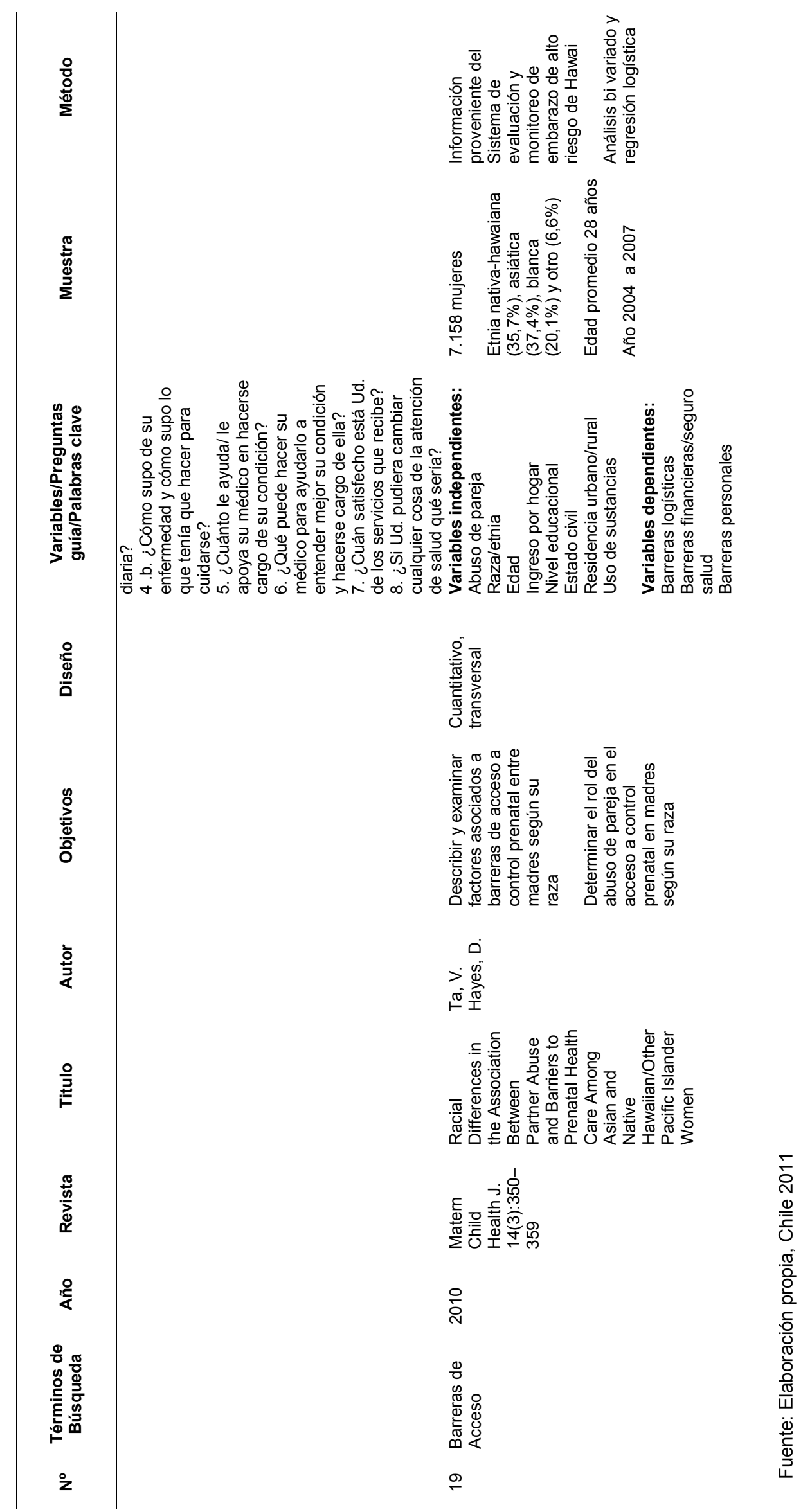




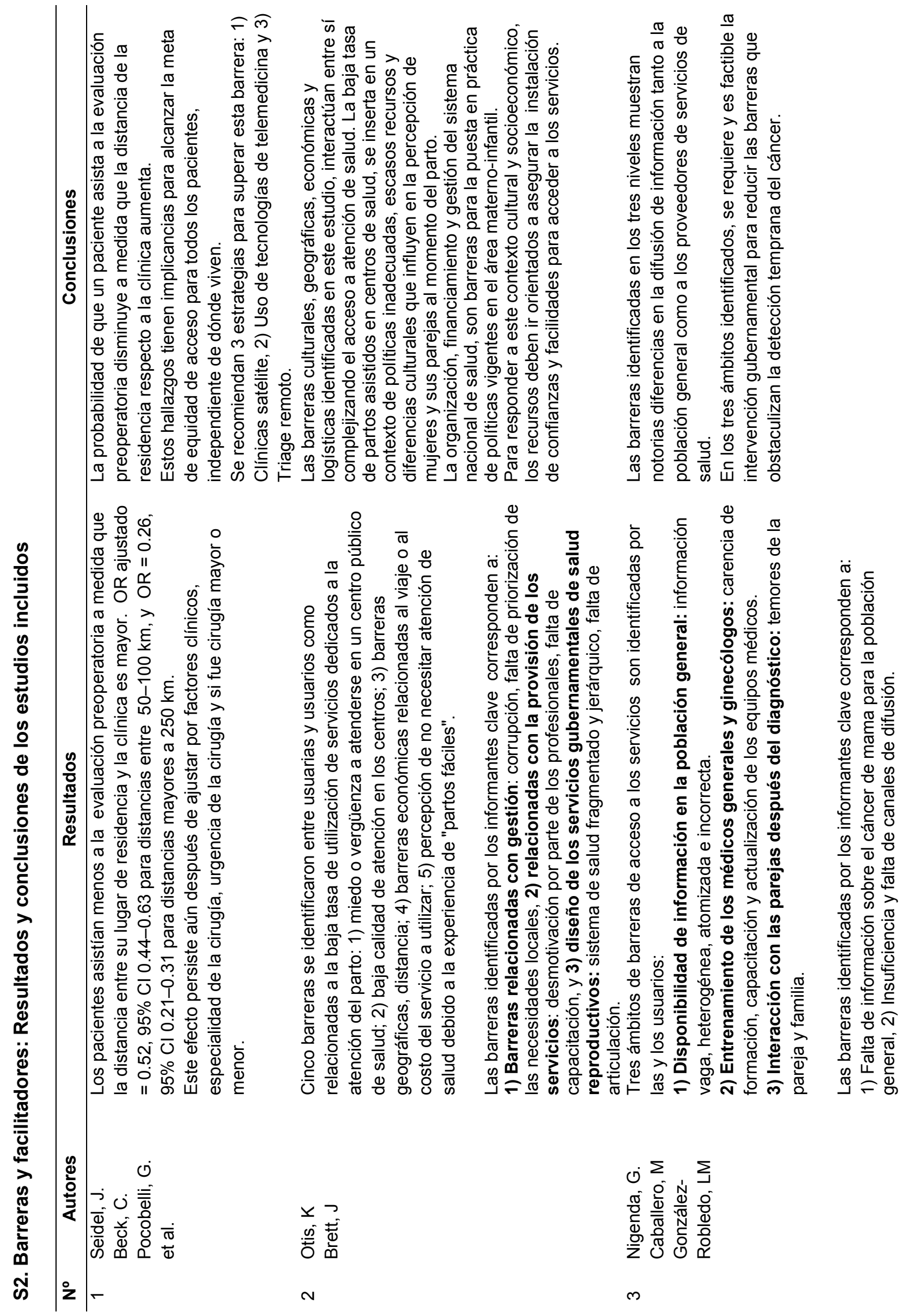




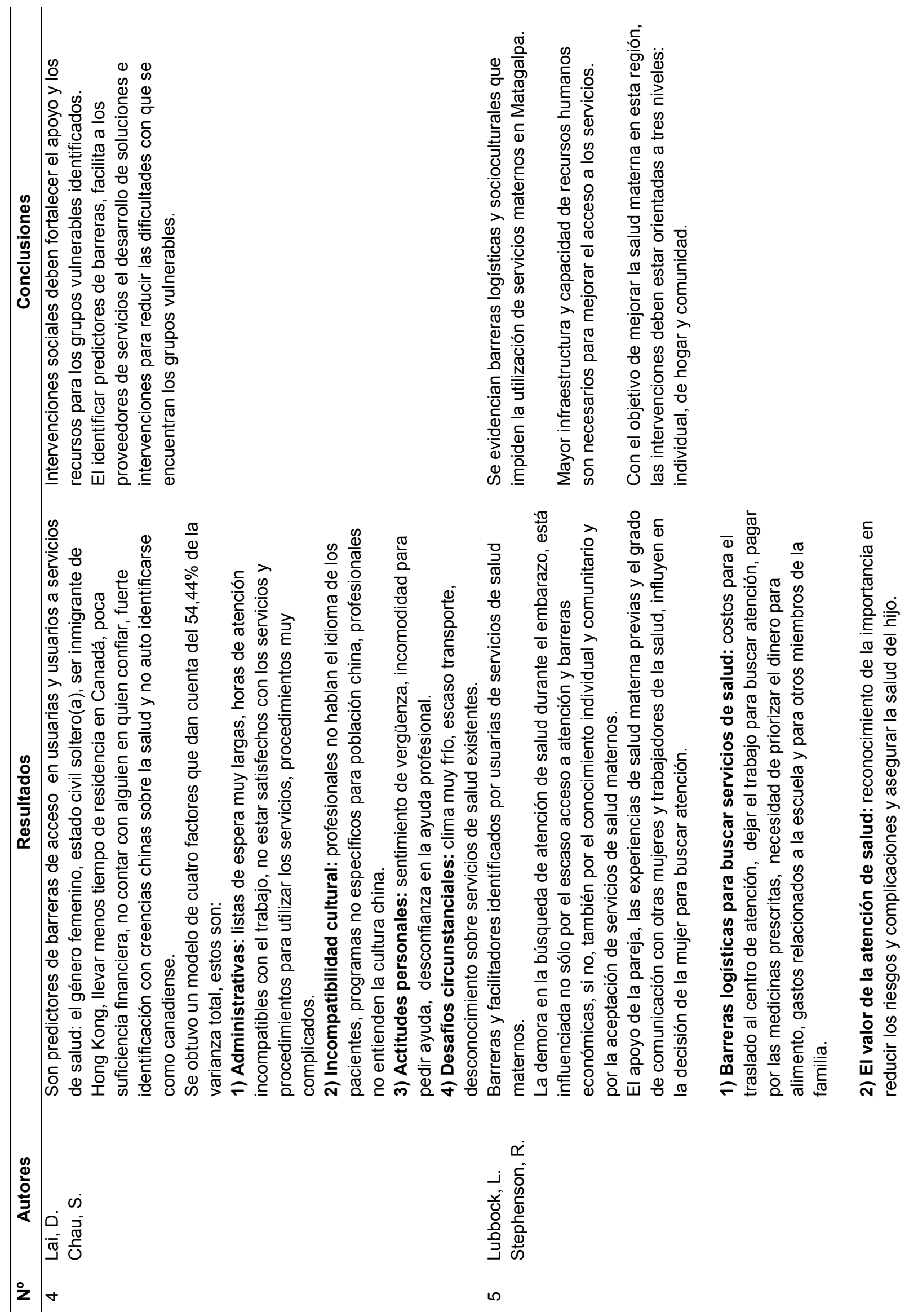




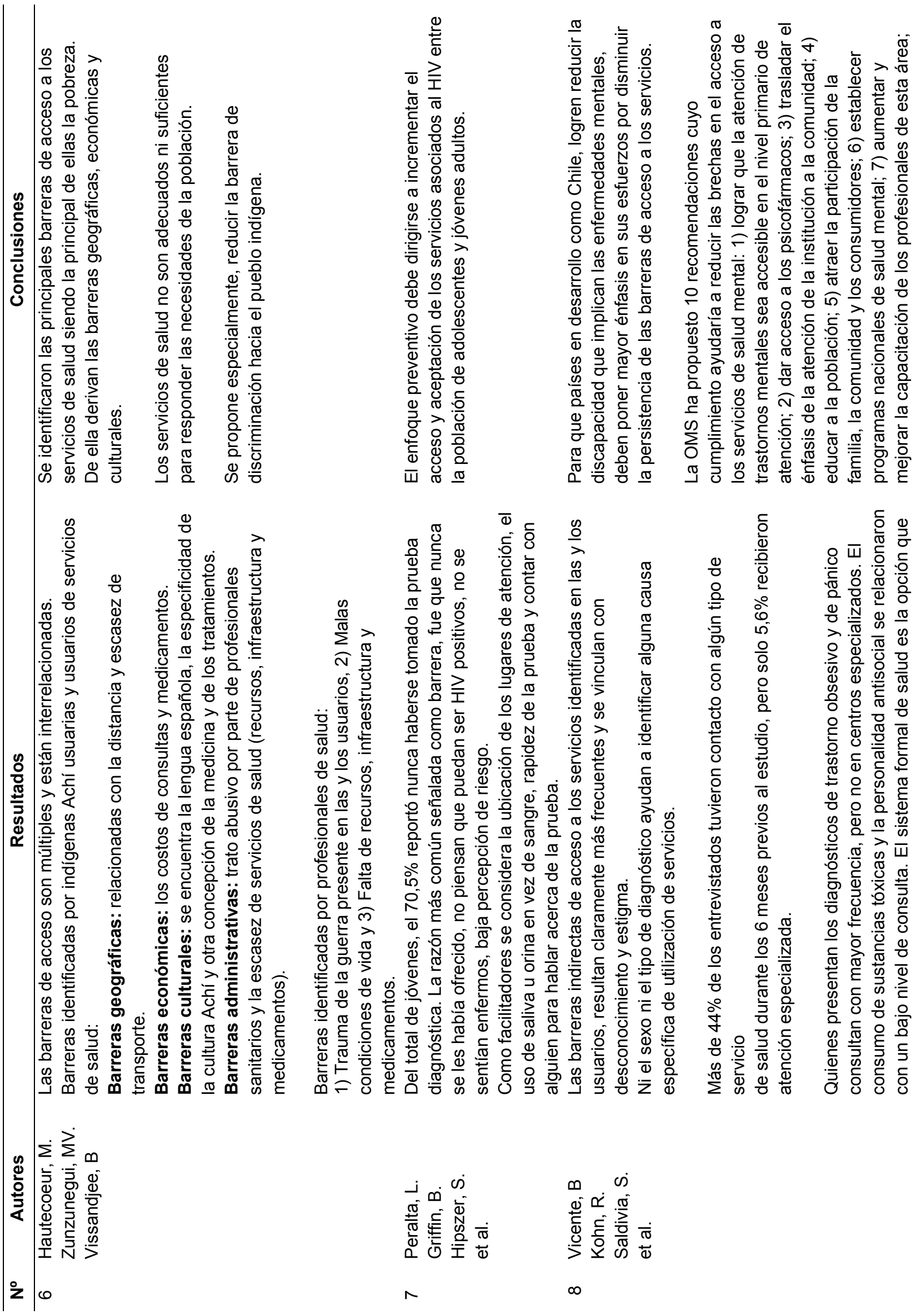



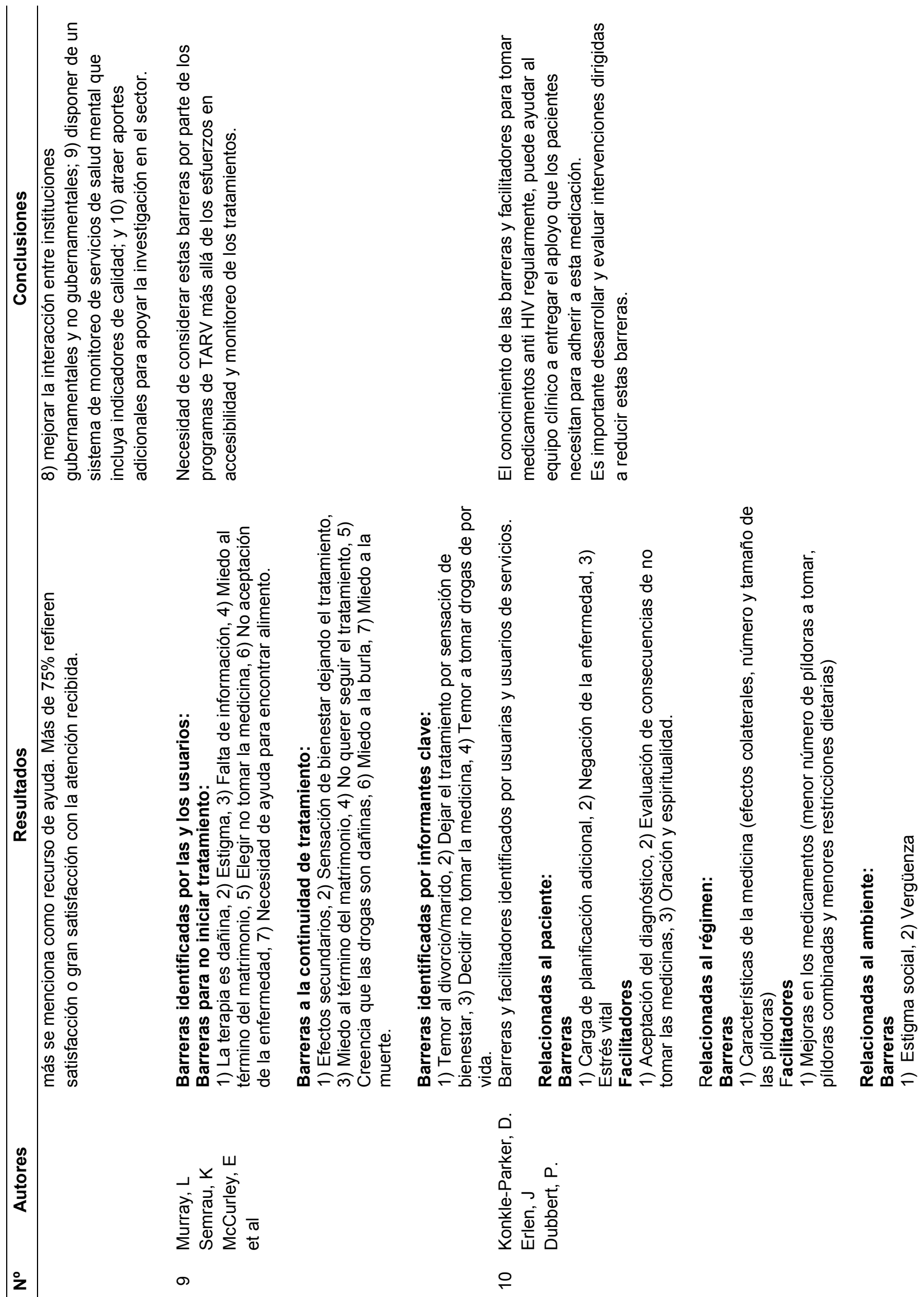

$\stackrel{\circ}{\circ}$ 

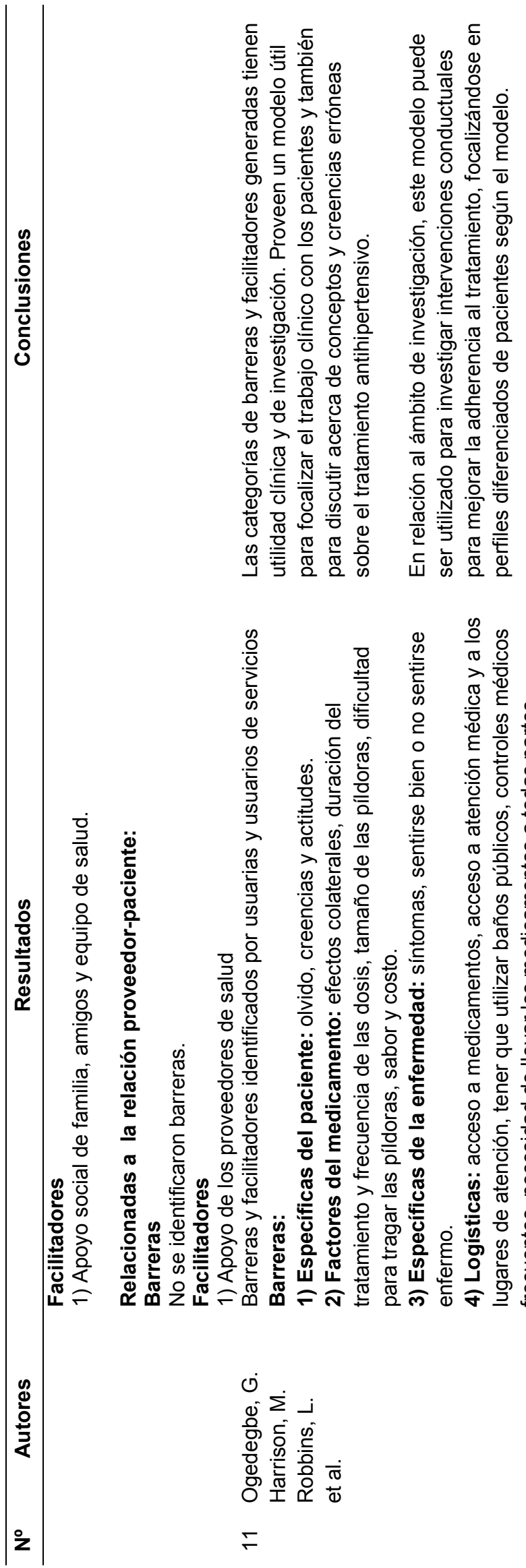

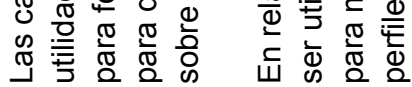
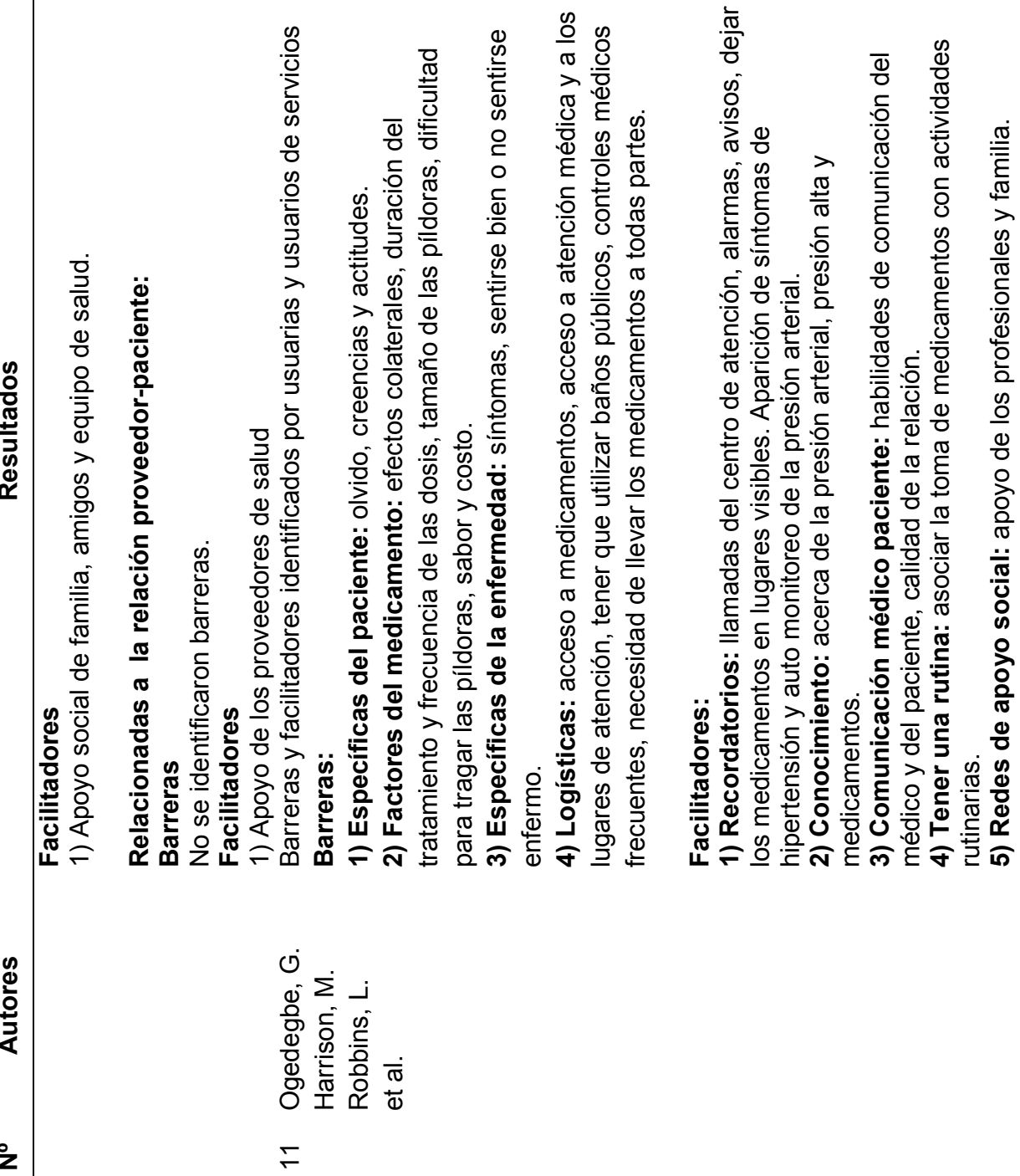


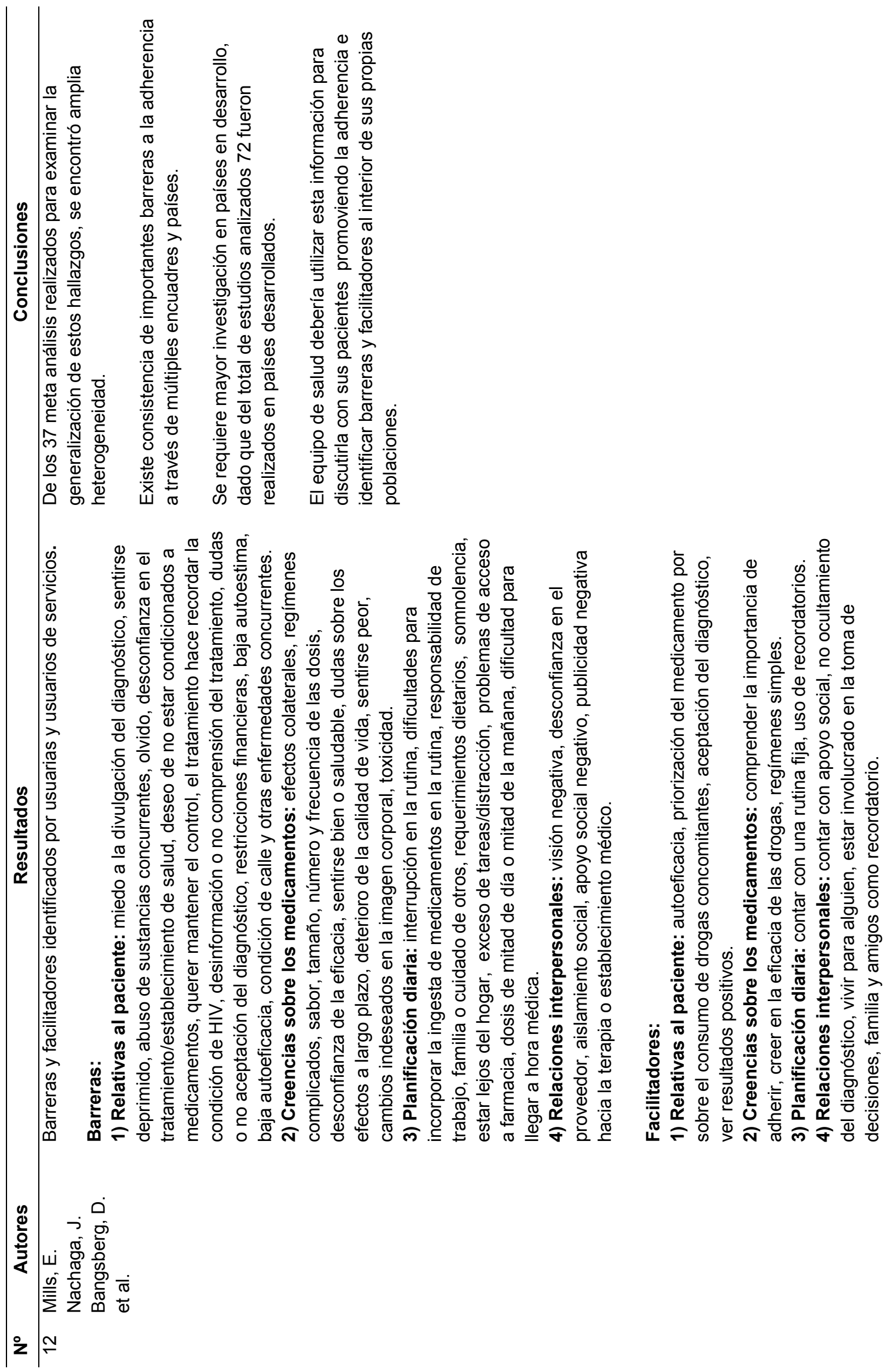



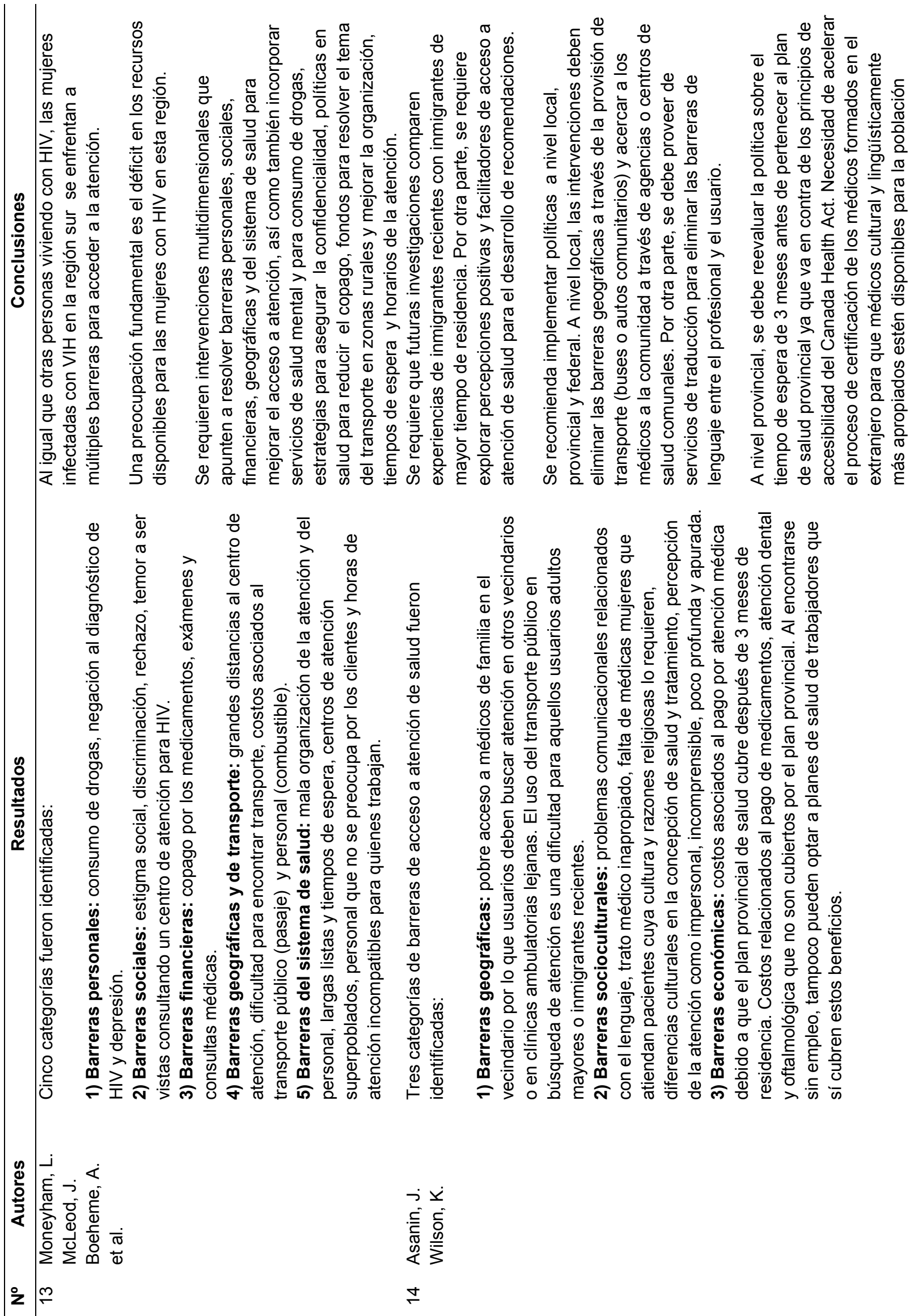

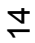



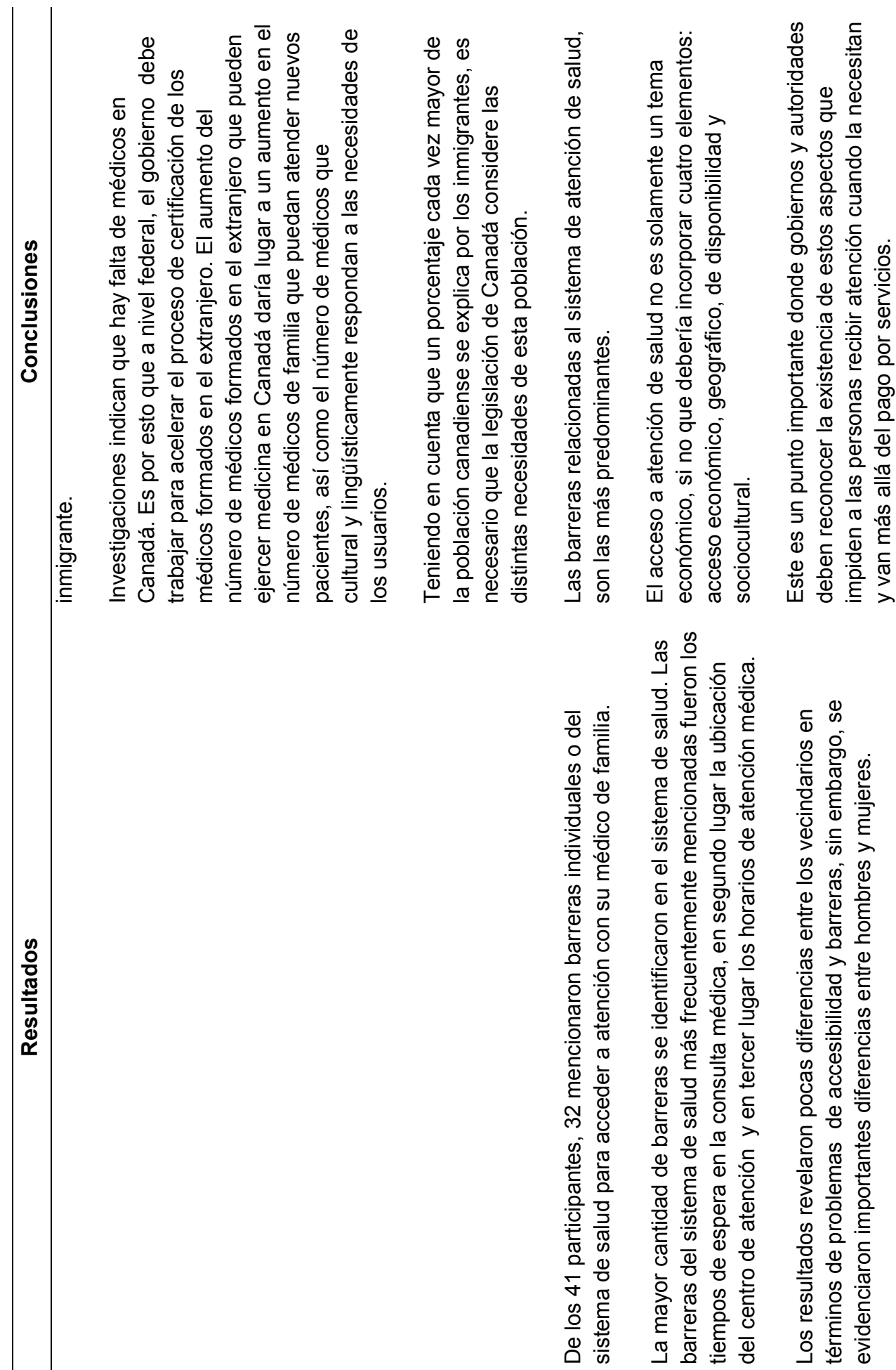

ڤั)

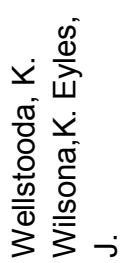

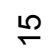




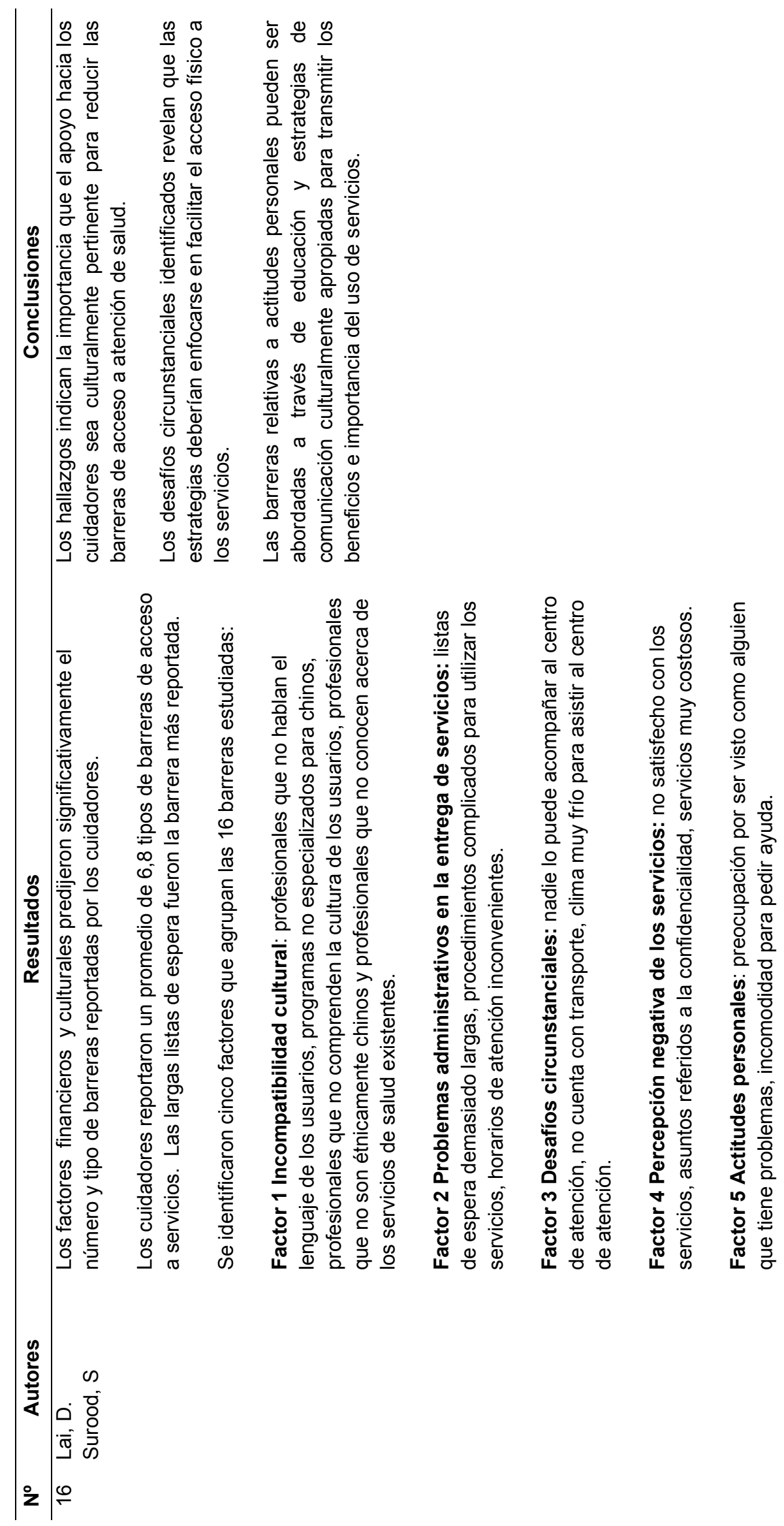



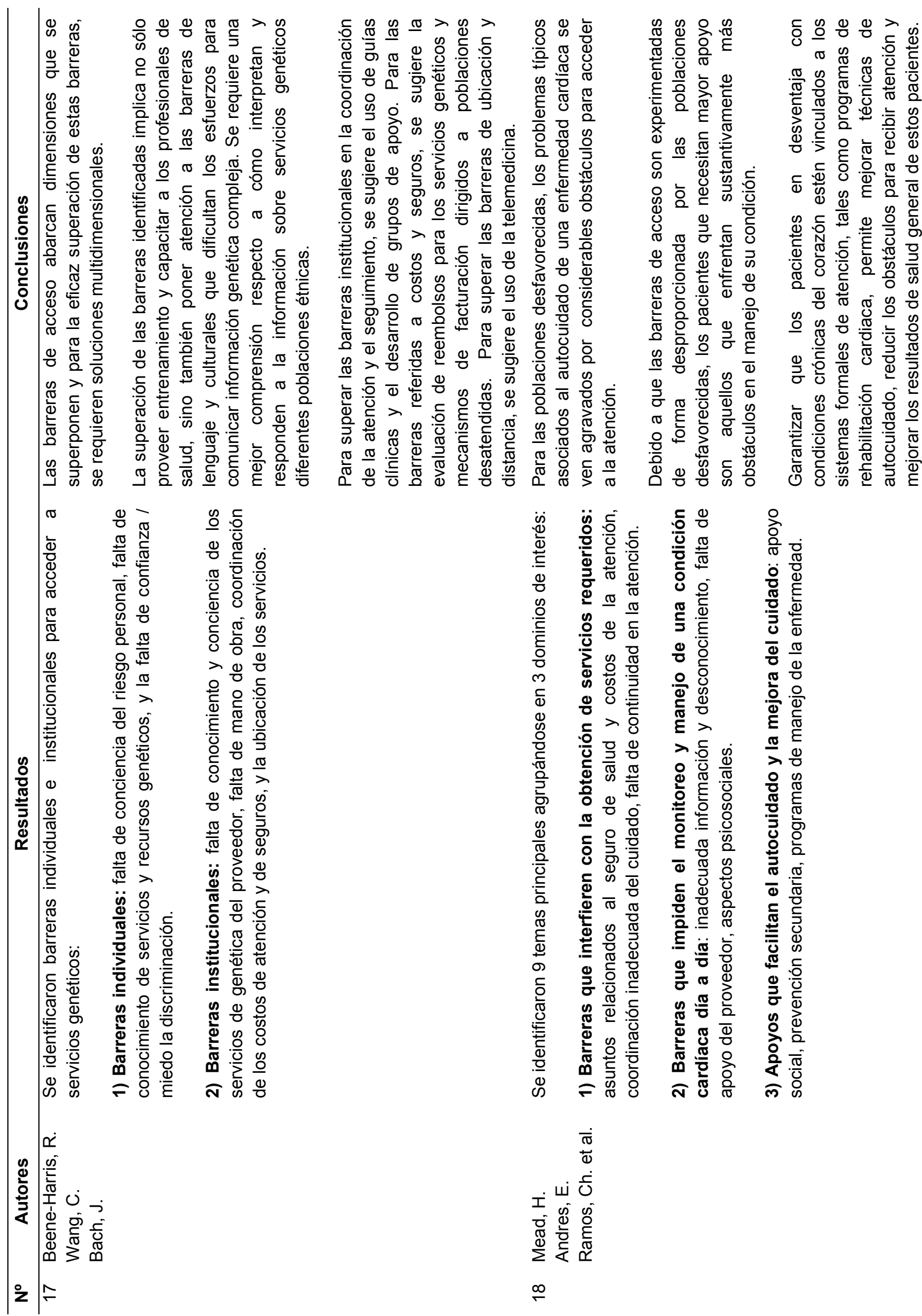


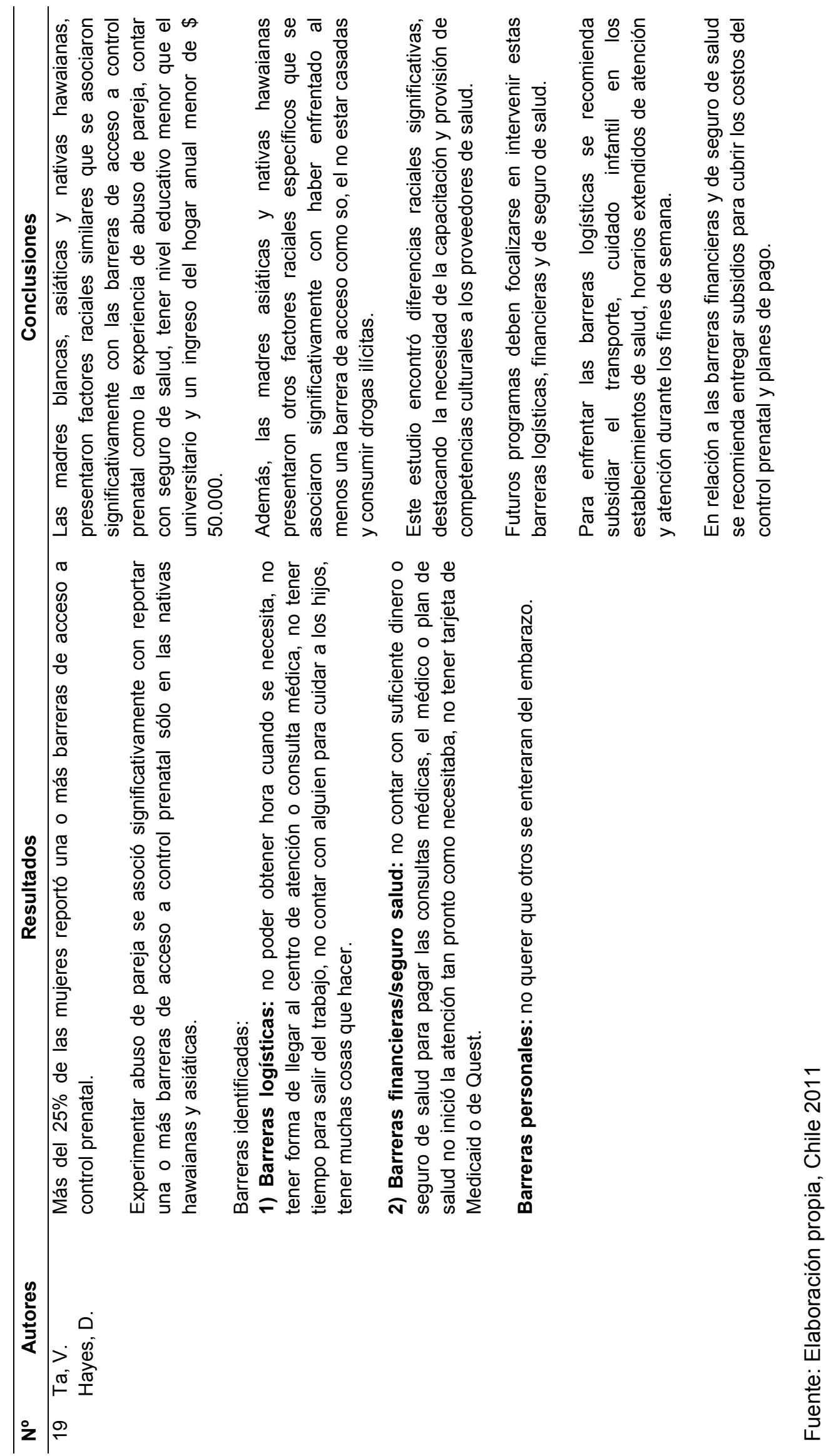




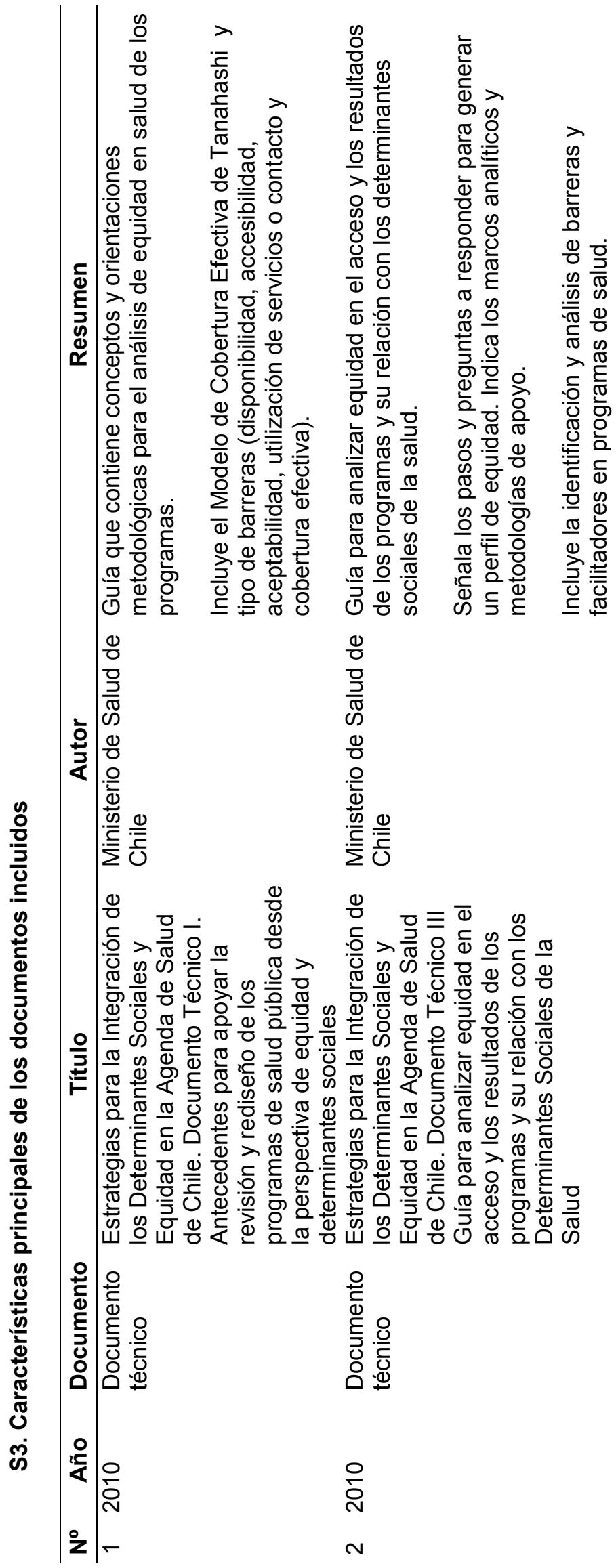




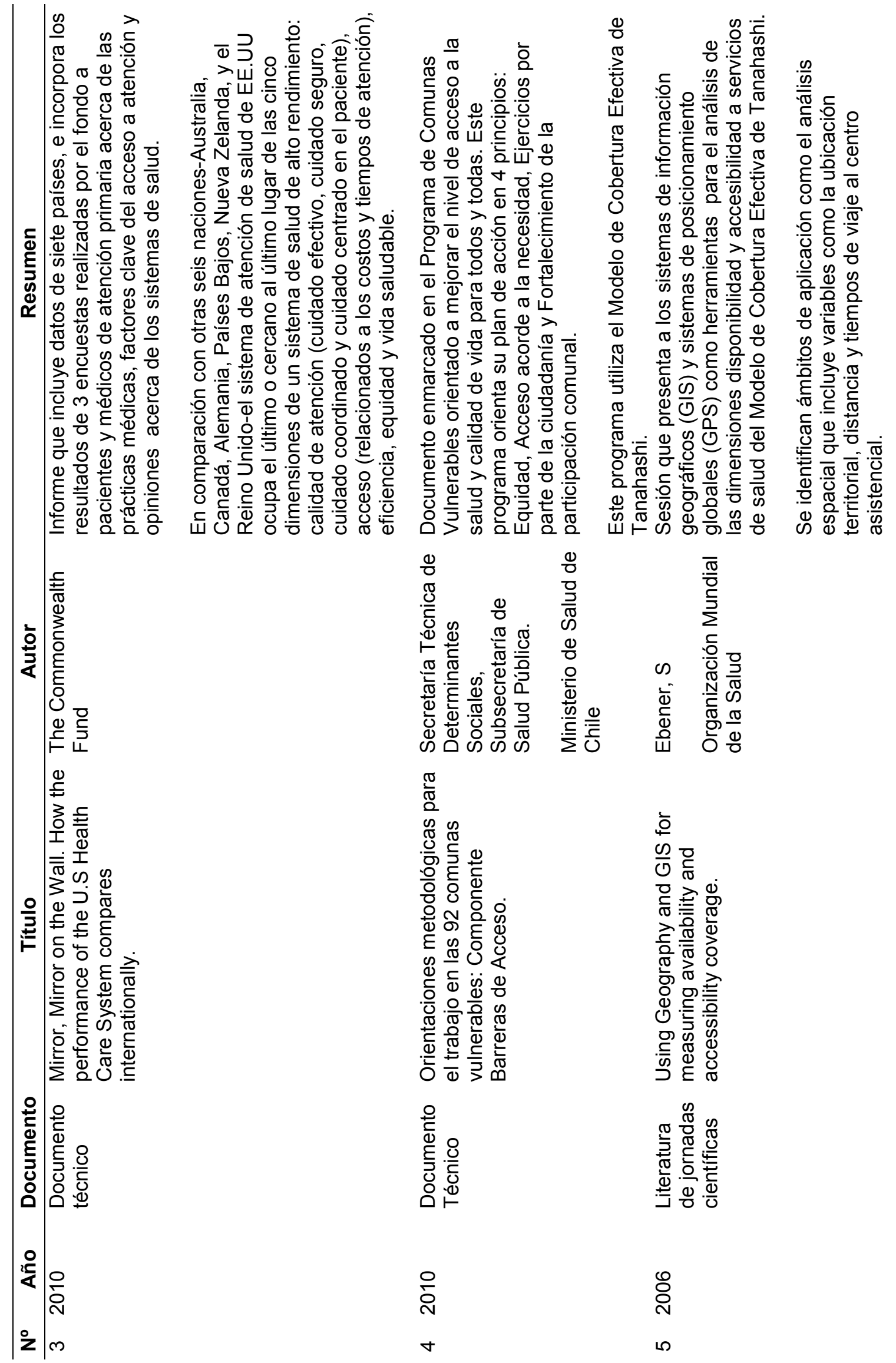




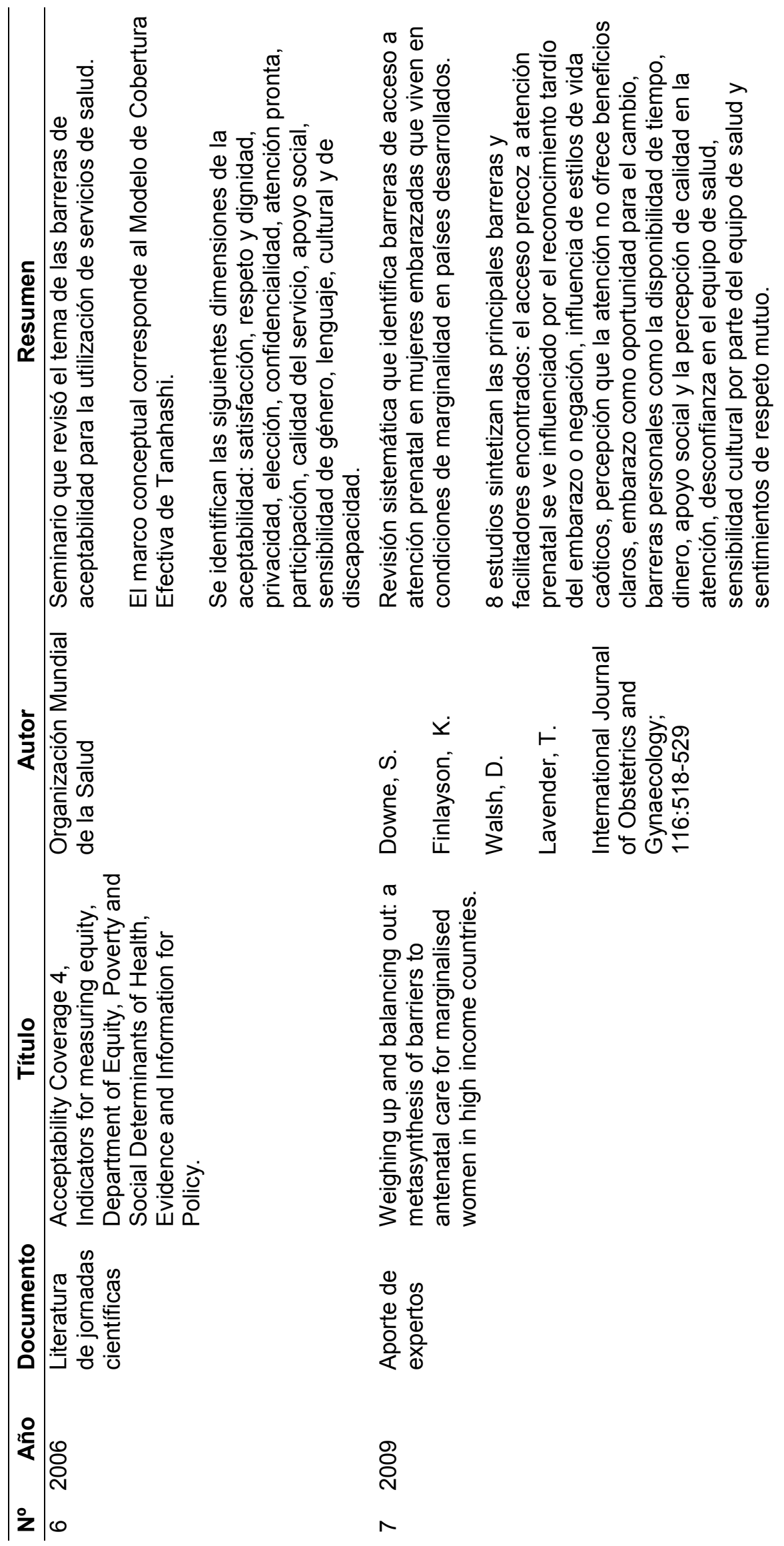




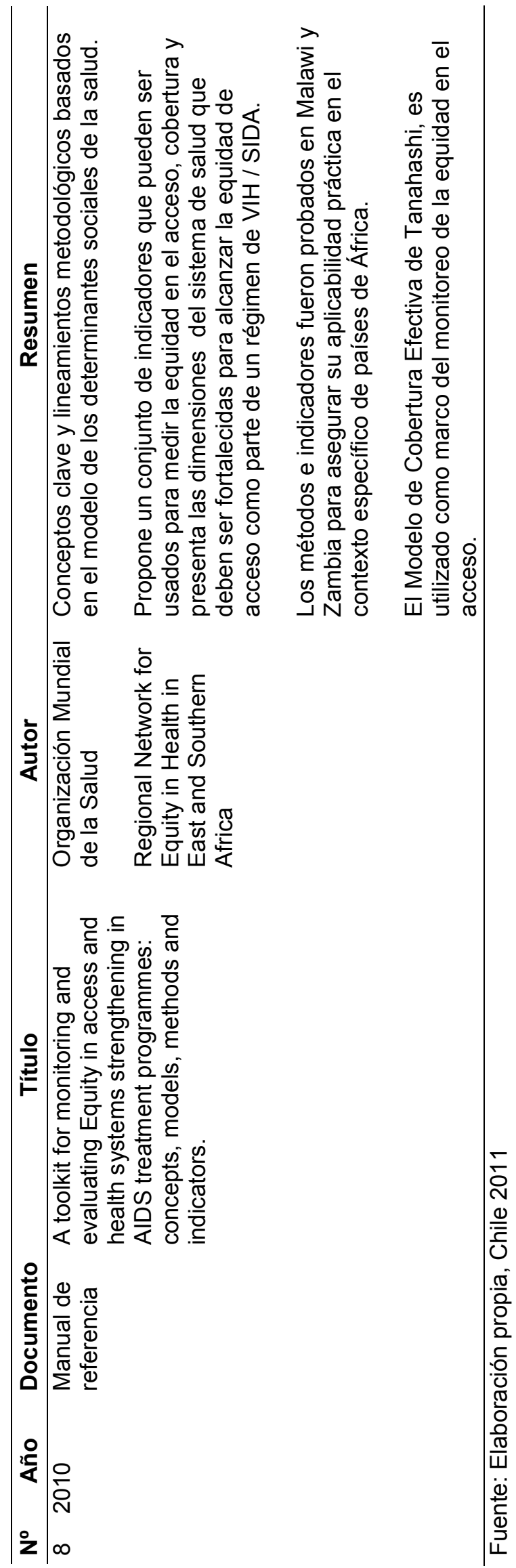

